\title{
Cortical Activation Patterns Evoked by Temporally Asymmetric Sounds and Their Modulation by Learning
}

\author{
Junsei Horikawa' ${ }^{1}$ and ${ }^{-}$Hisayuki Ojima ${ }^{2}$
}

\section{DOI:http://dx.doi.org/10.1523/ENEURO.0241-16.2017}

${ }^{1}$ Department of Computer Science and Engineering Graduate School of Engineering, Toyohashi University of Technology, Hibarigaoka 1-1, Tempaku, Toyohashi, Aichi 441-8580, Japan, ${ }^{2}$ Cognitive Neurobiology, Graduate School of Medical and Dental Sciences, Tokyo Medical and Dental University, 1-5-45 Yushima, Bunkyo-ku, Tokyo 113-8549, Japan

\section{Visual Abstract}

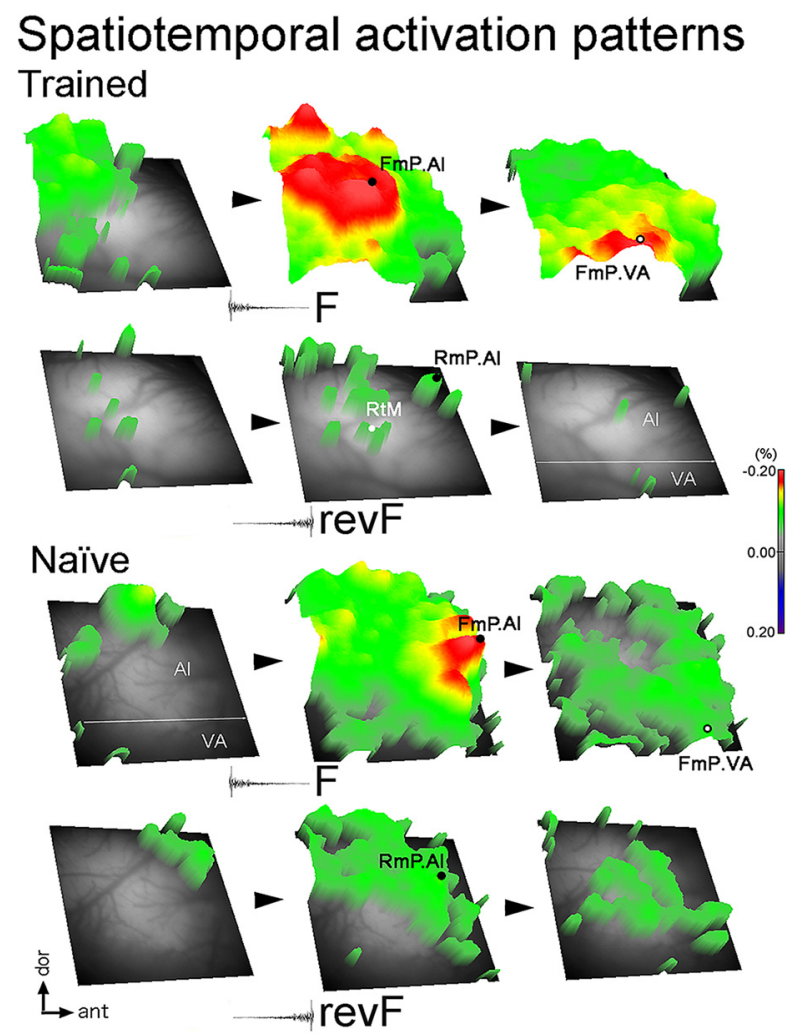

\section{Significance Statement}

Sound is perceived differently when it is played in the forward and reverse directions, despite that the duration and long-term spectrum profiles are identical for these time-reversed sounds. The perceptual differences must derive from the asymmetric processing of spectral transients of sounds and the temporal interactions of neuronal activity elicited by each transient. Spatiotemporal activation patterns can further our understanding of the temporally asymmetric sound processing. We for the first time imaged the distinct cortical activation evoked by a representative pair of temporally asymmetrical sounds and showed differences in the magnitude and location of the activation peaks and their latency from sound onset. Furthermore, sound recognition training enhanced neuronal activity in the belt field presumably involved in perceptual learning. 
When complex sounds are reversed in time, the original and reversed versions are perceived differently in spectral and temporal dimensions despite their identical duration and long-term spectrum-power profiles. Spatiotemporal activation patterns evoked by temporally asymmetric sound pairs demonstrate how the temporal envelope determines the readout of the spectrum. We examined the patterns of activation evoked by a temporally asymmetric sound pair in the primary auditory field (Al) of anesthetized guinea pigs and determined how discrimination training modified these patterns. Optical imaging using a voltage-sensitive dye revealed that a forward ramped-down natural sound $(F)$ consistently evoked much stronger responses than its time-reversed, ramped-up counterpart (revF). The spatiotemporal maximum peak (maxP) of F-evoked activation was always greater than that of revF-evoked activation, and these maxPs were significantly separated within the Al. Although discrimination training did not affect the absolute magnitude of these $\max P \mathrm{~s}$, the revF-to- $\mathrm{F}$ ratio of the activation peaks calculated at the location where hemispheres were maximally activated (i.e., F-evoked maxP) was significantly smaller in the trained group. The F-evoked activation propagated across the Al along the temporal axis to the ventroanterior belt field (VA), with the local activation peak within the VA being significantly larger in the trained than in the naïve group. These results suggest that the innate network is more responsive to natural sounds of ramped-down envelopes than their time-reversed, unnatural sounds. The VA belt field activation might play an important role in emotional learning of sounds through its connections with amygdala.

Key words: belt field; primary auditory field; sound discrimination; spatiotemporal activation; time-reversed sound; voltage-sensitive dye imaging

\section{Introduction}

The temporal variation in the overall sound amplitude, known as the envelope, is one of the critical factors for determining the quality of sounds. Natural sounds are biologically meaningful when they are presented in the forward direction but may be perceived as unfamiliar or peculiar when played in reverse (Licklider and Miller, 1951). Temporally reversed sound pairs are identical in their composition of the long-term spectrum power (Patterson, 1994a,b), but they are perceived differently. For artificial sound pairs, including one with damped envelopes (i.e., starting with a quick attack followed by an exponential decay) and the other with ramped-up envelopes (i.e., starting with a gradual attack followed by a rapid decay; Patterson, 1994a,b), human listeners perceive carrier sinusoids more robustly in the ramped-up version than in the damped version. Similarly, ramped-up sounds are perceived higher in loudness (Irino and Pat-

Received August 15, 2016; accepted April 3, 2017; First published April 14, 2017.

The authors declare no competing financial interests.

Author contributions: H.O. designed research; J.H. and H.O. performed research; J.H. contributed unpublished reagents/analytic tools; J.H. and H.O. analyzed data; J.H. and H.O. wrote the paper.

This work was supported by Grants-in-Aid for Scientific Research (C) JP22500368, JP26430034, and JP15K00234 from Japan Society for the Promotion of Science.

Acknowledgements: We thank Mrs. T. Ishida, R. Numata, and K. Hayashi for participating in data capturing; Ms. M. Takahashi for temporary care of animals; and Dr. M. Taira for continuous encouragement. We would also like to express many thanks to reviewers and the reviewing editor for their critical but kind comments.

Correspondence should be addressed to Hisayuki Ojima, Cognitive Neurobiology, Graduate School of Medical and Dental Sciences, Tokyo Medical and Dental University, 1-5-45 Yushima, Bunkyo-ku, Tokyo 113-8549, Japan. Email: yojima.cnb@tmd.ac.jp.

DOI:http://dx.doi.org/10.1523/ENEURO.0241-16.2017

Copyright @ 2017 Horikawa and Ojima

This is an open-access article distributed under the terms of the Creative Commons Attribution 4.0 International license, which permits unrestricted use, distribution and reproduction in any medium provided that the original work is properly attributed. terson, 1996) and longer in subjective duration (Schlauch et al., 2001). Comparable perceptual differences have been reported for sounds with broadband noises as a carrier (Akeroyd and Patterson, 1995). Previous reports also suggest that there is a minimal duration necessary for the temporal reversal of portions of a spoken sentence to deteriorate its intelligibility (Saberi and Perrott, 1999).

Behaviorally, previous studies have shown the asymmetric perception of ramped-down and ramped-up sounds in animals (Fay et al., 1996; Le Prell and Moody, 2000; Ghazanfar et al., 2001; Ojima and Horikawa, 2016). Rhesus monkeys showed right-ear orienting behavior to conspecific calls, whereas they exhibited left-ear orienting behavior to the time-reversed versions (Ghazanfar et al., 2001). This finding suggests a hemispheric lateralization of the processing of asymmetric sounds. In accordance with behavioral asymmetry, neuronal response preferences for species-specific calls over their time-reversed versions have been revealed in several animal species, including bats (Esser et al., 1997; Medvedev and Kanwal, 2008), songbirds (Margoliash, 1983; Doupe and Konishi, 1991), cats (Gehr, et al., 2000), and monkeys. A larger number of neurons in the marmoset primary auditory field (Al) preferred natural twitter calls than their time-reversed versions (Wang et al., 1995; Wang and Kadia, 2001). In rhesus monkeys, modest asymmetric response preferences were also found for several major vocalizations (Recanzone, 2008).

Except for vocalizations and calls, natural sounds are generally broadband noises and nonharmonic in spectral structure, and their instantaneous spectrum content varies rapidly. These sounds have been largely excluded from research interests, despite their prevalence in our environment. We can easily think of examples, such as knocking on a door, clapping hands, dropping hard objects, and stepping on the floor. The amplitude of these environmental sounds tends to be damped after the initial attack. Thus, the acoustic environment in nature may be biased toward the generation of damped type sounds 
rather than ramped-up type sounds. These environmental sounds are meaningful when they are played in the normal direction but less so if they are played in the reverse direction. We hypothesized that there should be corresponding biases in the neuronal mechanisms and connectivity for processing such time-reversed sound pairs. To test this hypothesis, we needed to record neural activity globally within the auditory cortex. Therefore, optical imaging was used to examine differences in responses to forward and reversed sound pairs. Considering the identical long-term spectrum content of the temporally asymmetric sounds pairs, we assume that potential asymmetric organization revealed will provide a clue to understanding how the temporal structure is influences the representation of the instantaneous spectrum of complex sounds and evolves over time as determined by the sound spectrogram.

The spatiotemporal pattern of neuronal activities is a fundamental readout of signals processed by sensory cortices (Cohen et al., 1978; Chemla and Chavane, 2010). A voltage-sensitive dye imaging technique can determine a spatiotemporal sequence of activation at a high temporal resolution. Using this technique and a temporally asymmetric sound pair, we aimed at answering the following questions. How different are the spatiotemporal activation patterns evoked by sound pairs with temporally asymmetric envelopes? Can discrimination learning modulate this putative differential processing?

\section{Materials and Methods}

\section{Behavioral experiment}

Animals

This study was approved by the local animal care committees at the universities for which both authors worked (nos. 0150209A and 26.7). It is also in accordance with the National Institutes of Health Guide for the Care and Use of Laboratory Animals (NIH publications no. 80-23, revised in 1996) as well as the policies of the Society for Neuroscience. Guinea pigs (Hartley, male; body weight, 350$400 \mathrm{~g}$ ) were purchased from a commercial supplier (Japan SLC). The animals were first transported to the university of one of the authors for training and then transferred to the university of the other author for imaging experiments. Naîve animals used as the control group were directly transported to the latter institute.

\section{Training, training facilities, and sound delivery system}

Guinea pigs were trained in a newly developed procedure in which competition was introduced to raise the motivation of animals (Ojima et al., 2012). During the training period, diet was strictly controlled for several days to maintain their body weight at $\sim 90 \%$ of the weight on the day of transportation. The body weight was then gradually increased. The training consisted of two stages. The early-training stage lasted for one week during which one pair of animals was housed in a single home cage within the laboratory. During this period, the animals were fed a small amount of pellets each time a conditioning sound was played through a dynamic speaker (NS-10MM, Yamaha). Feeding was arbitrarily timed within the sound-on period. After this acclimation period, the animals under- went daily training in which they were automatically fed at a constant delay of 1.6 or $3.2 \mathrm{~s}$ after the offset of a conditioning sound. The late-training stage lasted for another one week. During the first 3-4 d of this stage, the cage-mate pair was placed in a training arena $(50 \times 50$ $\mathrm{cm}$ ) within a sound-attenuating chamber and then trained with the training sound set (see below for details). During the remaining $3 \mathrm{~d}$, the paired animals were separately trained in an otherwise similar manner to the preceding days. On the next day following the late training, each animal was individually subjected to a one-chance trial of generalization testing (see below for details). Naïve animals (control group) were not trained, being housed for one or two weeks in a cage in an acoustic environment similar to the one used for the behavioral training. The husbandry staff was careful to not produce sounds similar to the conditioning sound while feeding or caring for the naïve animals.

The sound-attenuated chamber contained a training arena made of metal mesh (acoustically transparent), one monitor microphone (F-320, Sony), three video cameras (WAT-204CX, Watec and SH-6C, WTW), one custommade infrared motion detector, and two loudspeakers (NS-10MM, Yamaha) that were positioned $1.7 \mathrm{~m}$ above the arena and $1 \mathrm{~m}$ from each other. The loudspeakers were used for sound reproduction. The sound delivery system was calibrated $30 \mathrm{~cm}$ above a food saucer using a half-inch condenser microphone (7012, ACO) and compensated at 1/3-octave intervals from $80 \mathrm{~Hz}$ to $12.5 \mathrm{kHz}$ (Q2031B, Yamaha). Sounds were reproduced at $68 \pm$ 6-dB SPL after amplification through a power amplifier (N220, Sony).

\section{Training sounds and testing procedures}

The sounds used for training and testing were produced with a specialized software (Amadeus Pro, http:// www.hairersoft.com/) by editing natural sounds that were originally generated by stepping on the laboratory floor, clapping hands, hitting a plastic cage, hitting a metal can, scratching a metal mesh, and jingling keys. One representative sound segment from each of these natural sounds was duplicated 9-12 times to make respective multi-segment sound trains. The ramped-down forward natural sound $(F)$ used for conditioning was a footstep sound. Specifically, the conditioning sound was $4.7 \mathrm{~s}$ in duration and composed of $10 \mathrm{~F}$ segments with an identical spectrum except for the amplitude, which varied within a range of $\pm 6 \mathrm{~dB}$. The intersegment intervals were fixed to a relatively long value of $\sim 0.5 \mathrm{~s}$. Each $\mathrm{F}$ segment (110 $\mathrm{ms}$ in duration) had a ramped-down envelope with its peak pressure at $5 \mathrm{~ms}$ soon after sound onset and a spectral bandwidth extending up to $\sim 12 \mathrm{kHz}$. Much of its energy was distributed in the lower frequency region (Fig. 1). Other sounds were constructed in a similar way and used as distracters (nontarget sounds, NTs). The training sound set, which was played several times per day, consisted of six different sounds, including one $\mathrm{F}$ train and five NT trains (intersound intervals of $\sim 60 \mathrm{~s}$ ), presented in a randomized order for different trials, sessions, days, and animals. For the behavioral testing and imaging experiments, each segment of the F stimulus train was simply 


\section{A}
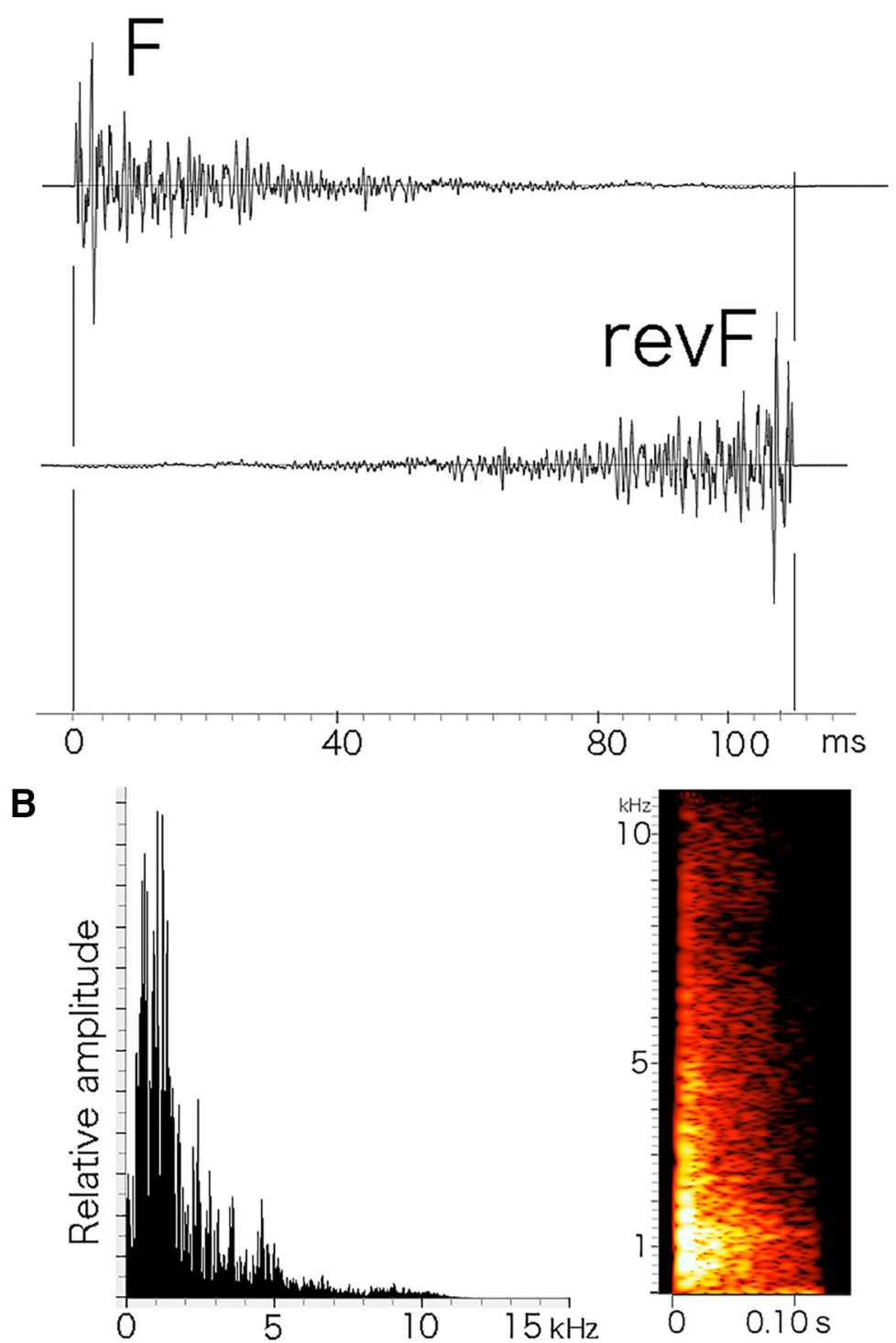

Figure 1. The stimulus sound segments, $F$ and revF, used for behavioral training and optical imaging. Sound stimuli are presented to animals as a train of four-time repeated $\mathrm{F}$ or revF segments in the optical imaging. $\boldsymbol{A}$, The $\mathrm{F}$ segment is a normal natural sound (footstep sound) and the revF segment is its time-reversed version. B, The power spectrum (left) and sonogram (right) of the $F$ segment. Note that the F and revF segments have an identical long-term power spectrum according to the Fourier transformation (Patterson, 1994a,b).

reversed in time to make the time-reversed version of $\mathrm{F}$ (revF; Fig. 1).

\section{Optical imaging experiment \\ Surgery}

Imaging was conducted from both hemispheres. After completion of imaging from one hemisphere, the second craniotomy was started for the other hemisphere. Animals were initially anesthetized with a mixture of ketamine and xylazine (i.m., 80 mg/kg, Ketalar, Daiichi-Sankyo, and 25 $\mathrm{mg} / \mathrm{kg}$, Selactar, Bayer Yakuhin, respectively), placed under a measuring microscope in a soundproof room, and artificially ventilated after injection of the muscle relaxant pancuronium bromide (i.m., $1 \mathrm{mg} / \mathrm{kg}$, Myoblock, MSD).
The auditory cortex exposed by craniotomy was stained with the voltage-sensitive dye RH795 (Invitrogen) for 60-90 min. Heart rate and body temperature were continuously monitored. A supplemental dose of anesthetics (25 mg/kg Ketalar and $10 \mathrm{mg} / \mathrm{kg}$ Selactar) and the muscle relaxant (1 mg/kg Myoblock) was administered every 60 $120 \mathrm{~min}$. Animals were euthanized with pentobarbital (i.p., $60 \mathrm{mg} / \mathrm{kg}$, Somnopentyl, Abbott) or ketamine (intracardiac, $160 \mathrm{mg} / \mathrm{kg}$ ) after completion of the experiment.

\section{Optical imaging procedure}

The auditory cortex contralateral to sound stimulation was epi-illuminated by a 480- to 580-nm fluorescent light with the ipsilateral ear clogged (Horikawa et al., 1996). Light signals 

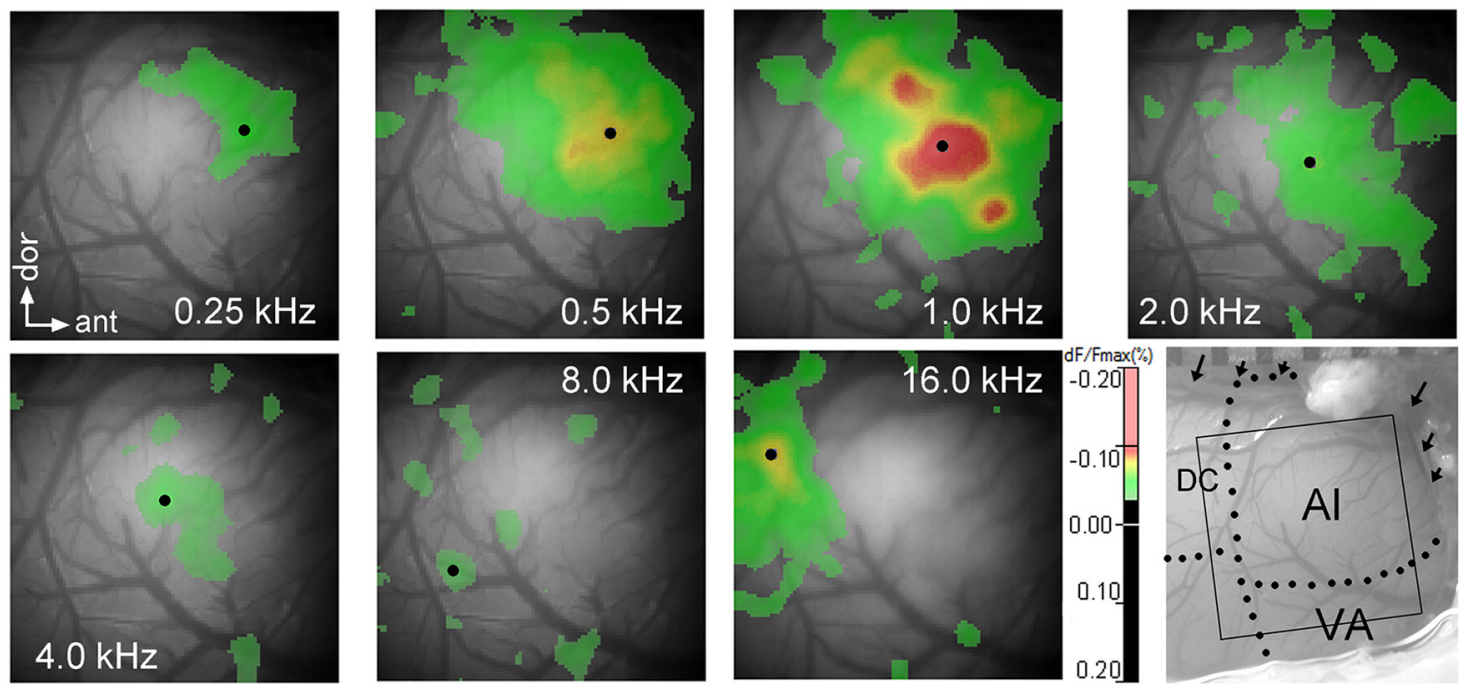

Figure 2. Tonotopic activation of the primary auditory cortex by a set of pure tones. Pure tones with a 200-ms duration and 5-ms onset/offset cosine ramps are reproduced at frequencies of $0.25,0.5,1,2,4,8$, and $16 \mathrm{kHz}$ at 75 -dB SPL in otherwise the similar manner to the asymmetric sound pair. On a conventional light micrograph (lower right), covering the anterior part of the guinea pig's $\mathrm{Al}$, the optical image frame (square) is superimposed. The approximate borders between the Al and DC field and those between the core Al and the belt VA are depicted by dotted lines. Thick blood vessels (a set of arrows) course along the pseudosylvian sulcus. Dots point to the maxima of activation evoked by different tones. For the tone-evoked activation maps, refer to Figure 3 .

emitted from the cortex (>620 nm) were recorded with a CMOS camera (MiCAM Ultima, www.brainvision.co.jp) attached to the measuring microscope. The camera consisted of a sensor array of $100 \times 100$ channels, which corresponded to a $5 \times 5 \mathrm{~mm}$ image frame. The image frame was captured every 2 ms with focusing at $300 \mu \mathrm{m}$ below the cortical surface. The Al was positioned approximately at the center of the image frame (Fig. 2, lower right) according to the pseudosylvian sulcus.

Sensor-detected light signals may include false-positive noises caused by fine movement derived from pulsation. To eliminate this artifact, the onset of each of the sound-on and silence periods was phase-locked to the simultaneously recorded electrocardiogram (ECG). Then, differential signals, extracted by subtracting the signals recorded during the silence period from those recorded during the sound-on period for each presentation of the sound unit, were averaged across its four presentations to obtain trial-unique differential signals (dF). Signals recorded at the periphery of image frames were tended to be less accurate because of weak light emission from the curved surface of cortex. Therefore, signals obtained from the marginal zone equivalent to a five-channel width from each side of the image frame were excluded from quantitative analyses.

\section{Sound stimulation during optical imaging}

Sound stimulation was controlled with a digital sound generation system (System 3, www.tdt.com) on a PC platform computer. A stimulus unit ( $\sim 4 \mathrm{~s}$ in duration), which consisted of (1) the sound train (sound-on period, 2 s) and (2) the equal period of silence immediately after the sound-on period, was digitally generated. One sound train contained four segments of either $F$ or revF (intersegment intervals, $0.5 \mathrm{~s}$ ). Delays of the onset of the stimulus units were temporally adjusted so that the timing of the ampli- tude maxima of $\mathrm{F}$ and revF segments had the same latency from imaging onset; namely, the first $F$ segment started $110 \mathrm{~ms}$ after imaging onset, while the first revF segment started $10 \mathrm{~ms}$ after imaging onset. The stimulus unit was consecutively repeated four times for averaging.

Illumination was started 3-4 s earlier than the sound onset, and thus the illumination period per trial was $\sim 20 \mathrm{~s}$ in duration. For a given hemisphere, each of the two stimulus sound types, one containing $F$ segments and the other containing revF segments, was presented once at a silent and dark interval of $\sim 100 \mathrm{~s}$. This relatively short intertrial interval together with the relatively short illumination period minimized the signal attenuation caused by photobleaching. The presentation order of $\mathrm{F}$ and revF stimulus types were randomized from hemisphere to hemisphere and this also eliminated the possibility of time-lag-based differences in signal attenuation between the different stimulus types. Sounds were reproduced at an average of 75-dB SPL through a loudspeaker (MSP$5 \mathrm{~A}$, Yamaha) placed $10 \mathrm{~cm}$ from the orifice of the animal's external auditory meatus. The spectrum-power specification of the system was compensated to match between behavioral training and optical imaging.

\section{Signal processing}

For each channel $(x, y)$, the dF of the background image captured just before the imaging onset was normalized to the maximum light intensity (Fmax) of the background image in each trial. If this normalized base intensity at a given channel, $F(x, y)$, was larger than 0.25 , the differential signal calculated for this channel, $\mathrm{dF}(\mathrm{x}, \mathrm{y})$, was modified to a value of $\mathrm{dF}(\mathrm{x}, \mathrm{y}) / \mathrm{F}(\mathrm{x}, \mathrm{y})$ (designated as $\mathrm{dF} / \mathrm{Fmax}, \%)$ as an optical response signal. If the $F(x, y)$ was equal to or smaller than 0.25 , the $\mathrm{dF}(\mathrm{x}, \mathrm{y})$ was assigned a value of zero. The dF/Fmax was measured at all the channels of each of the consecutive image frames for a given trial. 
Thus, for any channel, the temporal change of the dF/ Fmax signal was defined as a continuous trace with its amplitude varying as a function of time (Fig. $3 A a, b)$. This signal trace for a given channel (or location) was peaked at a certain time after sound onset, and this peak was designated as the temporal maximum (tempM or $t M$ ). If there was more than one peak at different times, the earliest one was adopted for a given trial. The dF/Fmax measured for all the channels of each of the consecutive image frames was also used to construct a trial-unique sequence of activation maps (Figs. 4, 5). Note that depolarization takes negative $\mathrm{dF} / \mathrm{Fmax}$ values and are represented as upward deflection in the response traces.

\section{Cortical activation maps}

On each image frame, cortical activation was mapped (Fig. $3 A c, B, C$ ) on the basis of the magnitude of normalized differential signals (dF/Fmax) across all the channels. For mapping, only depolarization was depicted. First, the signals were spatially filtered with an algorithm of averaging $7 \times 7$ neighbors. The temporal trace of the signal at each channel was then temporally filtered at 4 and $40 \mathrm{~Hz}$ for high pass and low pass, respectively. The largest signal value within each image frame was chosen as a spatial peak, and the largest of these spatial peaks across all image frames per trial was designated as the maximum peak (maxP or $\mathrm{mP}$; Fig. 3, large dots). If there was more than one channel showing the same $\max P$ value, signal values of the channels surrounding each peak channel were separately averaged and the one with the largest mean was chosen as the peak. Thus, the maxP represents the trial-unique spatiotemporal maximum evoked by a particular stimulus type, and can be defined separately for each of distinct cortical fields.

A portion of the signal trace recorded for $100 \mathrm{~ms}$ before the onset of the first $F$ segment reflects the period of spontaneous activity. Signal values of every 2-ms bin within this period of trace recorded at the $\max P$ location were averaged to obtain the spontaneous activation level for a given hemisphere. The 6 SD of this mean was used as the threshold to illustrate both the F- and revF-evoked activation maps, in which the suprathreshold channels were color-coded depending on their signal magnitudes. They were then superimposed on a black-and-white cortical surface image as shown in Figure 3 . A set of pure tones was applied to reconstruct a coarse tonotopic map (Fig. 2), usually immediately after capturing the response signals to the asymmetric sound pair.

\section{Data processing}

\section{Behavioral data analysis}

Behavioral performance was assessed by the initiation of behavioral reactions (BhRs), i.e., distinctive conditioned motion characterized by a quick head swaying combined with neck extension at the food saucer and/or circling the food saucer. The BhRs were easily discriminable from spontaneous motion at the food saucer or movement toward the saucer based on the restlessness and quickness of the animal. Trials were defined as positive only if the two following criteria were fulfilled: (1) animals initiated the BhR during the sound train-on period, and (2) they continued the BhR throughout a period of time up to the feeding moment for the $\mathrm{F}$ trials or the corresponding time for the revF trials.

\section{Imaging data analysis}

Stimulation with the sound containing four segments as a stimulus train evoked time-locked deflections on a dF/ Fmax signal trace (Fig. 3Aa, asterisks). Response signals evoked by only the first segment of the stimulus trains were used for the present analyses. This eliminated potential stimulus adaptation effects and alleviated the gradual deterioration in the ECG-based artifact cancellation power. Calculations were made for the following purposes and subjected to statistical analyses (see statistical Table). (1) To evaluate effects of the temporal asymmetry of sound envelopes on the response strength, the trialunique maxPs of activation were compared between the paired sounds. They were also compared between the animal groups to assess learning effects. (2) To evaluate whether a neuronal population maximally activated by the forward sound was also activated maximally by its time-reversed version, the spatial separation between the maxPs evoked by the paired sounds was compared with the spontaneous separation between the 2 maxPs evoked by repeating the forward sound twice. (3) To evaluate effects of the temporal asymmetry of sound envelopes on the activity of a neuronal population, the tempM of activation evoked by the reverse sound (RtM) at the location where a hemisphere was maximally activated (i.e., Fevoked $\max P$ ) was normalized to this hemisphere-unique $\max P$. These normalized values (RtM at FmP) were compared between animal groups. (4) For the temporal shift of activation, the activation period during which the suprathreshold activation was evoked was sequentially divided into four phases, and the spatial activation maps representing each of these four phases were chosen to illustrate the temporal activation pattern. Since the ventroanterior belt field (VA) was activated during the last phase of the response period, the maxP of the VA was defined. For comparisons of the normalized VA activation, the ratios of the $\max P$ within the $\mathrm{VA}$ relative to the $\max P$ within the $\mathrm{Al}$ were calculated for individual hemispheres and compared between animal groups.

\section{Statistics}

The McNemar's test was used for nonparametric statistical comparisons between the number of animals exhibiting BhRs to the $\mathrm{F}$ and revF sound pair. For each hemisphere, a paired $t$ test was used to compare the magnitudes of optical response signals evoked by $F$ and revF. An $F$ test was used to evaluate the unevenness of the delay time from the sound onset to the $\max P$ between animal groups. The Welch's test was used to compare signal amplitudes between animal groups. To statistically determine if the position of F-evoked activation peaks was spatially distinct from the position of revF-evoked activation peaks, we used the Welch's test to compare the distance between these two peaks to the distance between the activation peaks obtained by repeating $F$ twice. The comparison of the normalized VA activation between different animal groups was tested with the 


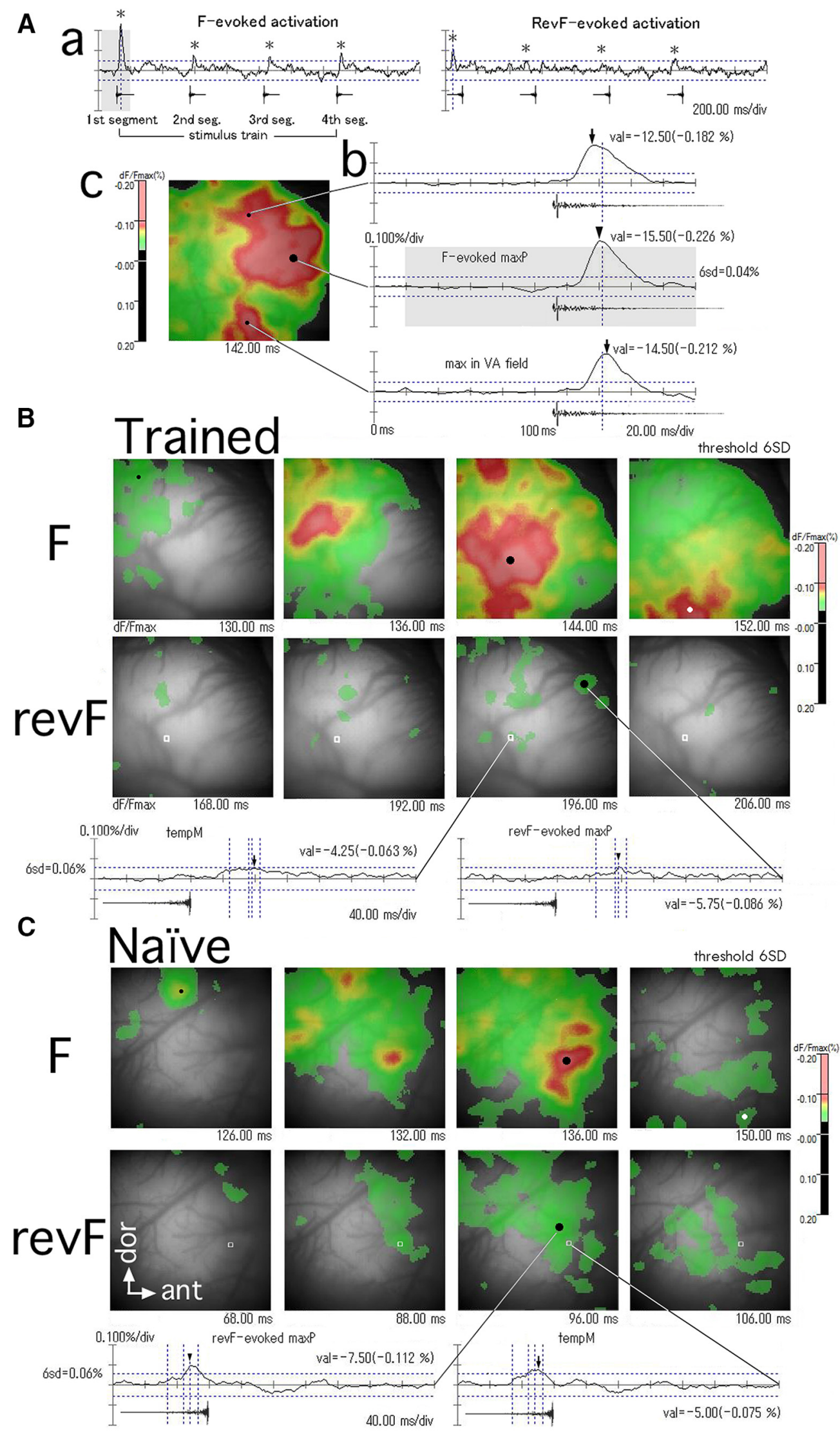

Figure 3. Temporal and spatial patterns of activation evoked by the temporally asymmetric sound pair. $\boldsymbol{A}$, Differential optical response signals (dF/Fmax, \%) recorded at single channels and an activation map generated from the signals recorded across all channels. 
continued

a, Traces of the response signals averaged across four-time repeats of the stimulus sound train which consists of four identical segments of either $\mathrm{F}$ (left) or revF (right), as shown below each response trace. Note that the F- and revF-evoked response traces are different even if they are recorded from the same channel. The temporal trace of responses typically shows 4 transient positive deflections that are time-locked to the individual sound segments (asterisks). Note that depolarization takes negative dF/Fmax values that are represented as the upward deflection in the response traces. The shaded portion of the response trace in $a$ is enlarged in the middle trace in $\boldsymbol{b}$. $\boldsymbol{b}$, Traces of the F-evoked response signals recorded at three different locations show the tempMs (arrows and arrowhead) in amplitude at different delay times after the sound onset. $\boldsymbol{c}$, A 2-ms image frame, recorded at the time of the dotted vertical line in $\boldsymbol{b}$, shows the map of activation that is above the threshold (i.e., 6 SD of the mean of spontaneous activities). The suprathreshold signals are color-coded according to their magnitude (scale bar). Each image frame has a spatial peak, and the largest of these peaks across all the frames recorded for a given trial is designated as the trial-unique maxP. The maxP of activation within the $\mathrm{Al}$ is indicated by the large dot in the map and corresponds to the peak (arrowhead) of the trace shown in $\boldsymbol{b}$. The time when the image frame is recorded (imaging onset is 0 ) is shown just below the activation map. Image frames have the dimensions of $5 \times 5 \mathrm{~mm}$. $\boldsymbol{B}, \boldsymbol{C}$, Temporal sequence of activation maps evoked by the first $\mathrm{F}$ and the first revF segments (upper and lower panels, respectively) during the period of activation in the trained $(\boldsymbol{B})$ and naïve $(\boldsymbol{C})$ animals. The $6 \mathrm{SD}$ of the mean of spontaneous activity values is used as the threshold. Large black dots indicate the maxPs within the Al. Small black dots in the F panels show the initial activation peak during the activation period. White dots in the $\mathrm{F}$ panels point to the maxP of F-evoked activation within the VA. Two temporal traces of the revF-evoked activation, one recorded at the channel of the revF-evoked maxP (large black dots in the revF panel) and the other recorded at the channel corresponding to the F-evoked $\max P$ (open white squares), are shown below traces. Arrowheads and arrows indicate the tempM at the respective recording channels (note that the arrowhead on the trace recorded at the large black dots corresponds to the revF-evoked maxP). Dotted vertical lines on the revF-evoked traces indicate the time when different image frames are recorded.

Welch's test. The number of hemispheres that showed peaked activation within the VA was compared between the animal groups using the Fisher's exact probability test.

\section{Results}

In this section, results of behavioral observations are described first. Following it, peak values of the response signals evoked by the $\mathrm{F}$ and its time-reversed counterpart (revF) under anesthesia are presented. Then, spatial activation patterns based on the separation of the trial-unique maxPs evoked by the $\mathrm{F}$ and revF within the $\mathrm{Al}$ are shown. For learning effects, the magnitudes of $\mathrm{F}$ - and revFevoked maxPs and their relative magnitudes (contrasts) are compared between the trained and naîve animal groups. Finally, for corticocortical propagation of peaked activation to the belt field, the relative magnitudes of the F-evoked $\max P$ within the VA to that within the Al are compared between the groups.

\section{Behavioral tests with the temporally reversed sound pair}

In the trained group, animals were conditioned to be rewarded on $\mathrm{F}$ sounds only. During the training period, especially in the late-training stage, it was common to observe conflict behaviors such as keeping their body over the food saucer to block the competitor's approach to it and/or inserting their snout at the orifice of the food hopper to interfere with competitor's food intake (Ojima and Horikawa, 2016). Such aggressive behavior was never observed for naïve animals. Behavioral tests showed that all of the trained subjects $(n=11)$ responded with BhRs to the $F$ stimulus train, but none of them responded to the revF train except for one subject (McNemar's test, $p=$ 0.002 ; Table 1). In contrast, naïve subjects $(n=11)$ did not respond to either stimulus sound train of $F$ or revF segments (McNemar's test, $p=1.0$; Table 1). These results indicate that the guinea pig can discriminate temporally asymmetric sound types.

\section{Identification of the $\mathrm{Al}$ and belt fields of the guinea pig}

Optical response signals were obtained from 12 hemispheres of 8 trained guinea pigs and 12 hemispheres of 6 naïve guinea pigs. These 24 hemispheres were used to compare the maxPs of Al activation. For the maxPs of VA activation, hemispheres that had bleeding locally from the dura mater near the ventral edge of the image frame were eliminated (18 hemispheres used). On the basis of the mirror-symmetric tonotopic organization of cortical activity to the pure tone set, the border between the $\mathrm{Al}$ and dorsocaudal cortex (DC) was estimated. The response latencies and temporal activation patterns were used to estimate the approximate border between the $\mathrm{Al}$ and VA (Wallace et al., 1999). In general, the ventral one-fifth zone of the image frame except for a narrow posterior zone (i.e., DC) corresponded to the VA (Fig. 2, lower right). Domains of the pure tone-evoked activation gradually shifted more posterior across the Al as the frequency increased, with the tendency for its anterior part to be activated more widely than its posterior part (Fig. 2). This may be consistent with the dominance of lower frequencies in the frequency spectrum of the guinea pig's communication calls (Berryman, 1976) and also with the overrepresentation of lower frequencies within the guinea pig's Al (Wallace and Palmer, 2009).

\section{Cortical activation evoked by $F$ and revF segments in the core field}

Optical response signals evoked by $F$

For the trained animal group, a single $F$ train sequentially evoked four sharp positive deflections on the signal trace at the activated channels (Fig. $3 A a$, left). The channel-unique tempM of activation evoked by the first $F$ segment was gradually delayed as recording sites were spatially shifting from the dorsal to ventral region (Fig. $3 A b)$. The delay time of the maxP of F-evoked activation after the onset of imaging (Fig. 6) was relatively constant 


\section{A $F$, Trained}
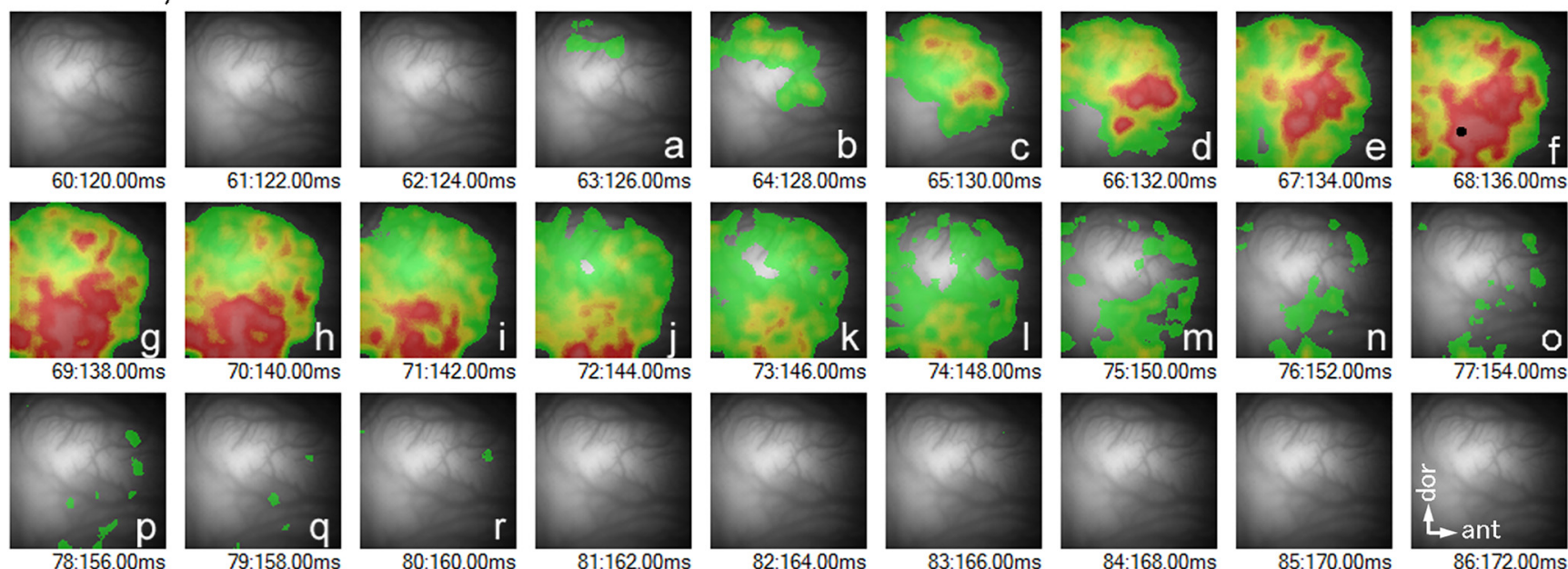

$0.100 \% /$ div
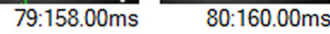

$81: 162.00 \mathrm{~ms}$
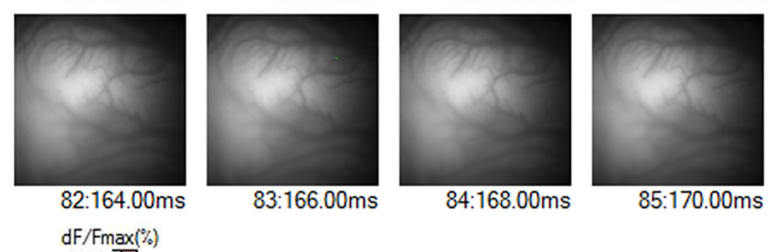

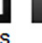

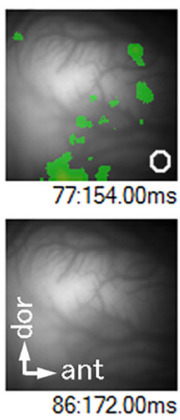

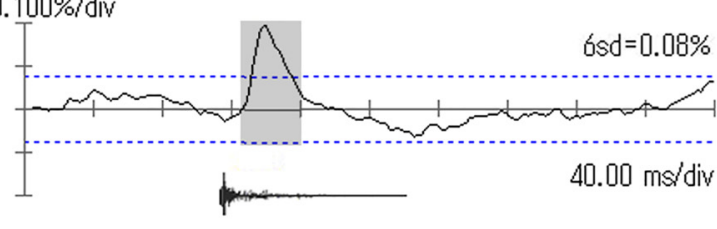

B revF, Trained

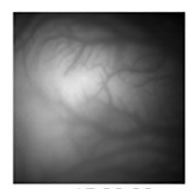

$15: 30.00 \mathrm{~ms}$

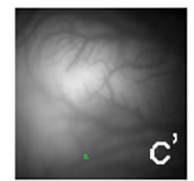

$24: 48.00 \mathrm{~ms}$

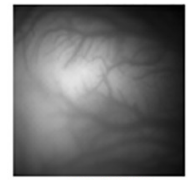

33:66.00ms

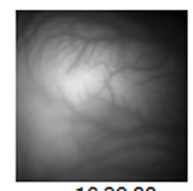

$16: 32.00 \mathrm{~ms}$

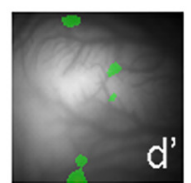

25:50.00ms

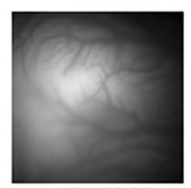

$34: 68.00 \mathrm{~ms}$

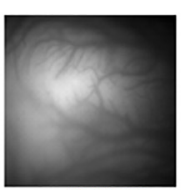

$17: 34.00 \mathrm{~ms}$

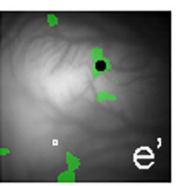

26.52.00ms

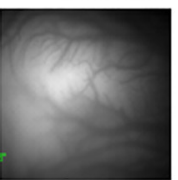

35:70.00ms
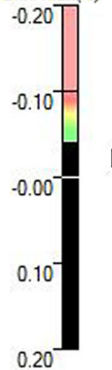

0.20

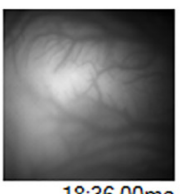

$18: 36.00 \mathrm{~ms}$

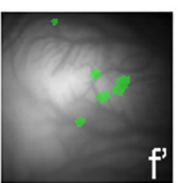

27:54.00ms

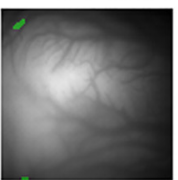

36:72.00m
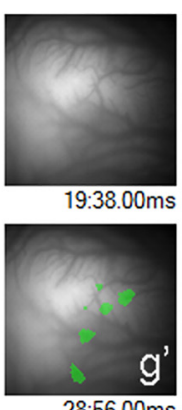

28:56.00ms

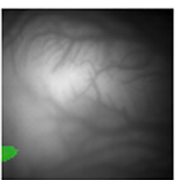

37:74.00ms

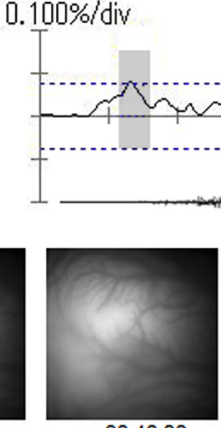

$20: 40.00 \mathrm{~ms}$

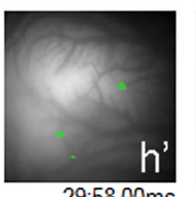

29:58.00ms

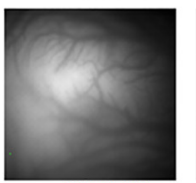

38:76.00ms

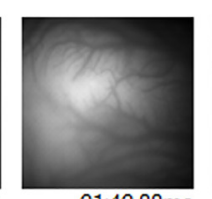

$21: 42.00 \mathrm{~ms}$

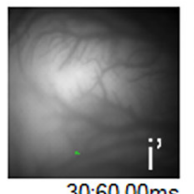

$30: 60.00 \mathrm{~ms}$

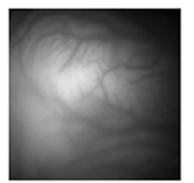

39:78.00m

Figure 4. Full-time course of trial-unique activation maps in the trained animal. The activation maps evoked by the 1st segments of $\mathrm{F}(\boldsymbol{A})$ and its time-reversed revF $(\boldsymbol{B})$ in a trained animal are chronologically arranged at 2-ms intervals. The temporal traces of response signals recorded at the F- and revF-evoked maxPs (large dots in $\boldsymbol{A} \boldsymbol{f}$ and $\boldsymbol{B e}^{\prime}$ ) are shown below or above the respective frame sequences. In the revF-evoked activation map, the location where the F-evoked maxP is evoked is indicated by the open white square $\left(\mathbf{B e}^{\prime}\right)$. The activation maps labeled with lower-case letters are derived from the hatched portions of the response traces. All image frames have the dimensions of $5 \times 5 \mathrm{~mm}$.

across different hemispheres $(24-42 \mathrm{~ms}, 28.3 \pm 5.2 \mathrm{~ms}$, SD; Fig. 7; Table 2).

A spatial activation map was generated from the signals of all channels of a single image frame at the threshold of $6 \mathrm{SD}$ of the spontaneous activity mean (Fig. 3Ac). As shown in the Figure $3 B$, upper panel, time course of activation evoked by the first $F$ segment demonstrates that the domains of activation was consistently evoked initially in the dorsal region of the $\mathrm{Al}$ (small black dot representing the local peak; Fig. $3 B$, upper panel, left frame; Nishimura et al., 2007). The peaked activation (red/pink domains) shifted ventrally/anteroventrally in a few tens of milliseconds along an axis roughly perpendicular to the anterocaudal frequency gradient as the major domain of activation increased its amplitude and extent across the Al. The trial-unique maxP (Fig. 3B, upper panel, 


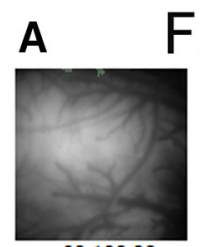

$60: 120.00 \mathrm{~ms}$

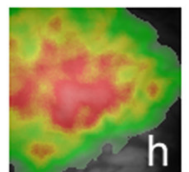

69:138.00ms

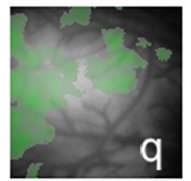

$78: 156.00 \mathrm{~ms}$ $0.100 \% / \mathrm{div}$

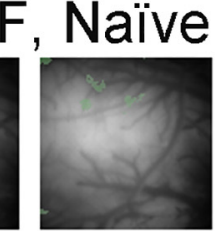

$61: 122.00 \mathrm{~ms}$

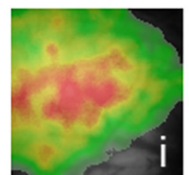

70:140.00ms

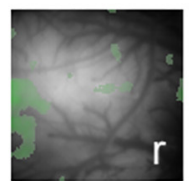

79:158.00ms

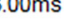

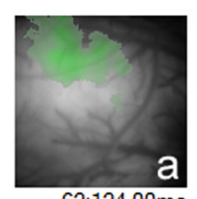

62:124.00ms

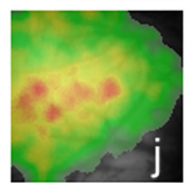

71:142.00ms

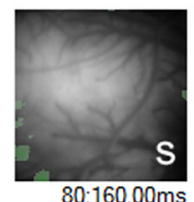

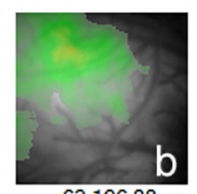

$63: 126.00 \mathrm{~ms}$

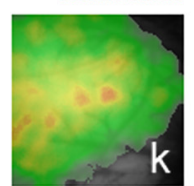

$72: 144.00 \mathrm{~ms}$
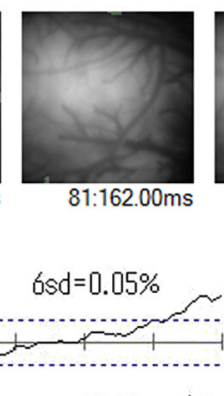

$40.00 \mathrm{~ms} / \mathrm{div}$
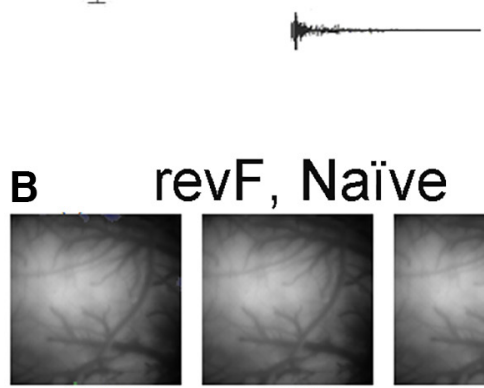

revF, Naïve

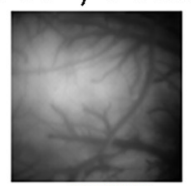

$15: 30.00 \mathrm{~ms}$

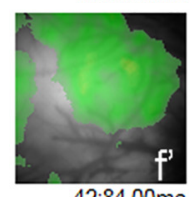

42:84.00ms

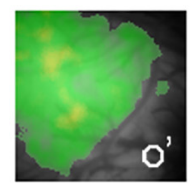

69:138.00ms

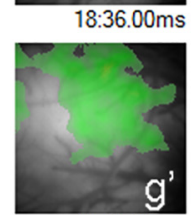

45:90.00ms

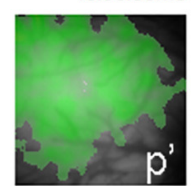

$72 \cdot 144.00 \mathrm{~ms}$
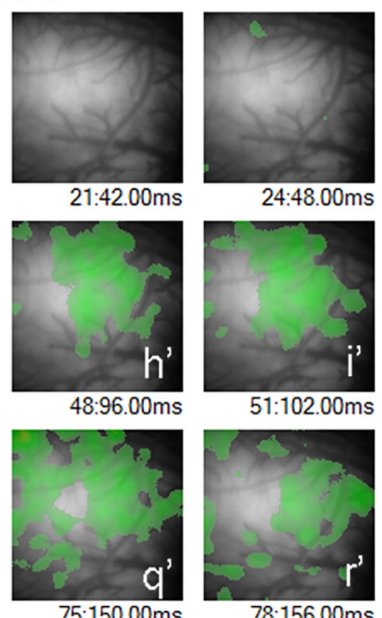
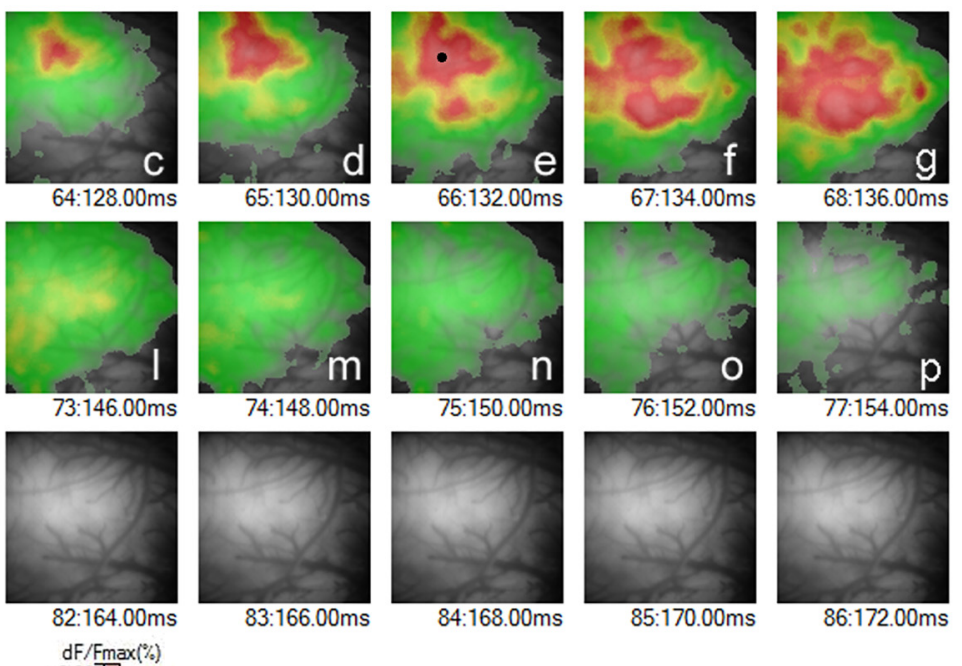

83:166.00ms

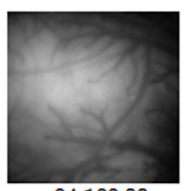

$84: 168.00 \mathrm{~ms}$

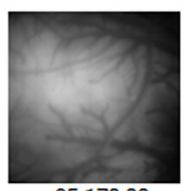

$85: 170.00 \mathrm{~ms}$
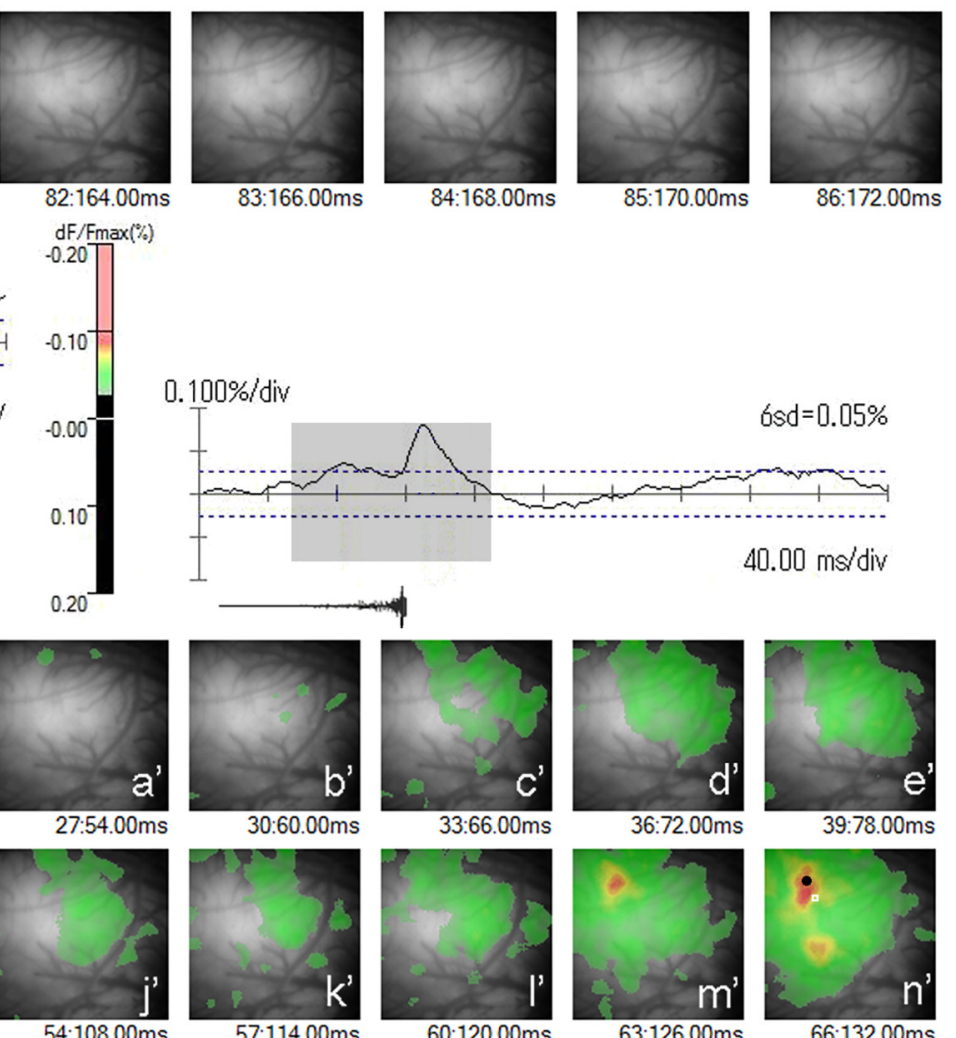

$0.100 \% /$ div

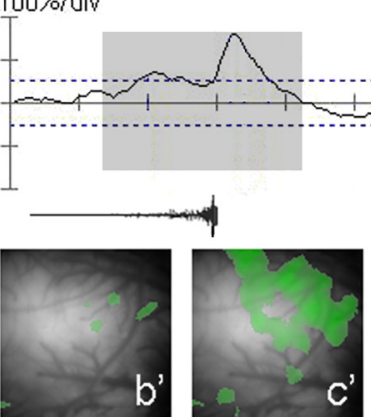

$30: 60.00 \mathrm{~ms}$
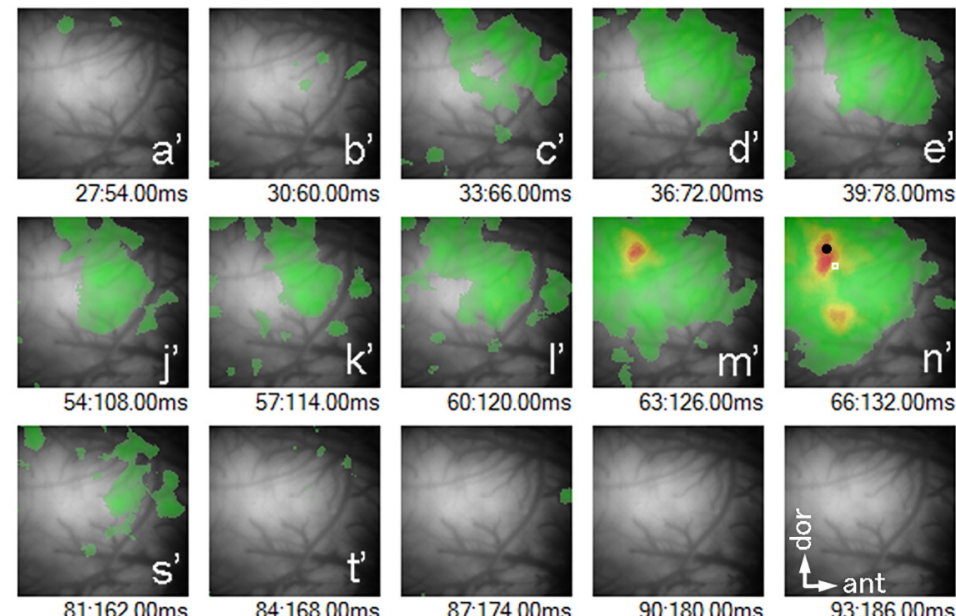

bsd $=0.05 \%$
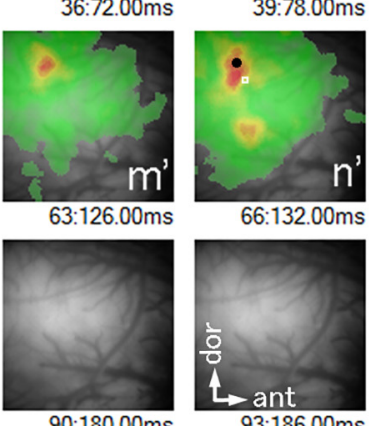

Figure 5. Full-time course of trial-unique activation maps in the naïve animal. The activation maps evoked by the 1st segments of $F$ $(\boldsymbol{A})$ and its time-reversed revF $(\boldsymbol{B})$ in a naïve animal are chronologically arranged at 2- and 6-ms intervals, respectively. The temporal traces of response signals recorded at the F- and revF-evoked maxPs (large dots in $\boldsymbol{A e}$ and $\mathbf{B n}^{\prime}$ ) are shown below or above the respective frame sequences. In the revF-evoked activation map, the location where the F-evoked max $P$ is evoked is indicated by the open white square $\left(\boldsymbol{B n}^{\text {') }}\right.$. The activation maps labeled with lower-case letters are derived from the hatched portions of the response traces. All image frames have the dimensions of $5 \times 5 \mathrm{~mm}$.

large black dots in the third frame) was typically found within the strongly activated domain in the Al. Interestingly, the peaked activation extended further ventrally/ anteroventrally and shifted to the field ventral to the Al (i.e., VA) in additional several milliseconds with little attenuation of its peak amplitude (Fig. 3B, upper panel, small white dot in the right frame). A complete temporal sequence of activation was shown for another hemisphere in Figure $4 A$. The similar patterns of F-evoked activation were observed for almost all hemispheres (Fig. 8, left column).

For the naïve animal group, the delay time of the $\max P$ of F-evoked activation (Fig. 6) was also constant across different hemispheres (22-34 ms, $26.3 \pm 4.5 \mathrm{~ms}$, SD; Fig. 7; Table 2), and not statistically different from the trained animal group (Welch's test, $p=0.323$; Table 2). As in the 
Table 1. Comparison between behavioral performances evoked by different sound types

\begin{tabular}{|c|c|c|c|c|c|c|}
\hline $\begin{array}{l}\text { Animal } \\
\text { group }\end{array}$ & $\begin{array}{l}\text { Number of } \\
\text { animals } \\
\text { used }\end{array}$ & $\begin{array}{l}\text { Response to both } \\
\mathrm{F} \text { and revF (number } \\
\text { of animals) }\end{array}$ & $\begin{array}{l}\text { Response to } \mathrm{F} \text { but } \\
\text { not to revF (number } \\
\text { of animals) }\end{array}$ & $\begin{array}{l}\text { Response to revF but } \\
\text { not to } F \text { (number } \\
\text { of animals) }\end{array}$ & $\begin{array}{l}\text { Response to neither } \\
\mathrm{F} \text { nor revF (number } \\
\text { of animals) }\end{array}$ & $\begin{array}{l}\text { Comparison between } \\
\text { sound types }\end{array}$ \\
\hline & & & & & & Type of test \\
\hline Trained & 11 & 1 & 10 & 0 & 0 & McNemar test 0.00195 \\
\hline Naïve & 11 & 0 & 0 & 0 & 11 & McNemar test 1.000 \\
\hline
\end{tabular}
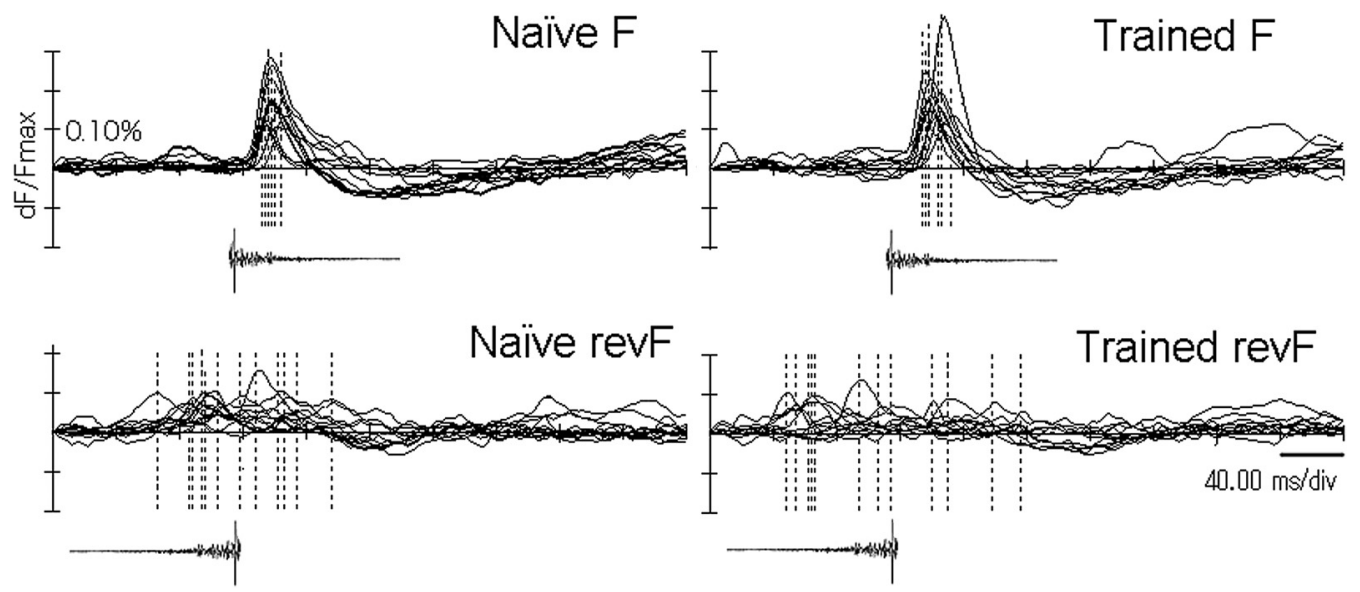

Figure 6. Trial-unique response traces recorded at the maxP. Temporal traces of the response signals (dF/Fmax, \%) evoked by the first $F$ segment at the channels where the spatiotemporal maxP within the Al is evoked. The sound waveforms below traces show the delay times and duration of the stimulus sounds ( $F$ and revF). The time of maxP is indicated by the dotted line for each trace. $F$, normal natural sound. revF, time-reversed version of $F$.
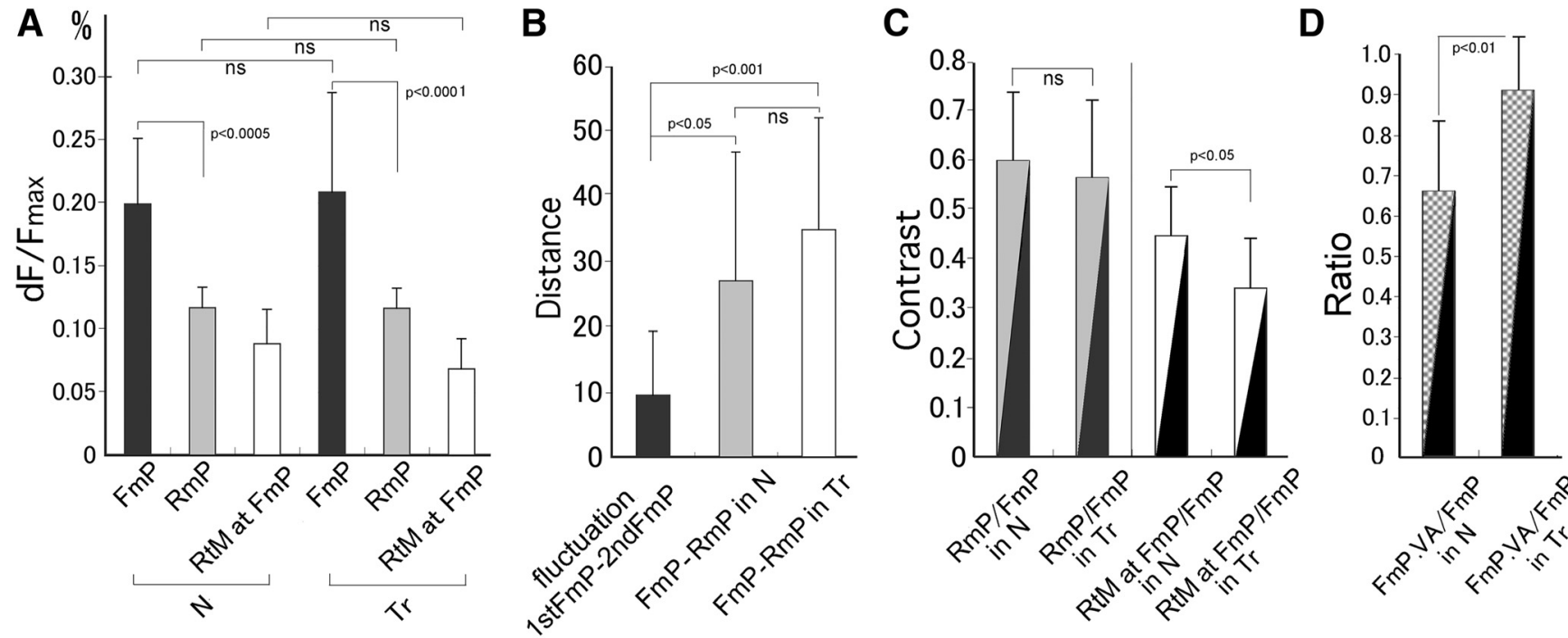

Figure 7. Quantitative comparisons of the peaks of sound-evoked signals between the $F$ and revF stimulation and between the animal groups. $\boldsymbol{A}$, The trial-unique maxPs of activation within the $\mathrm{Al}(\mathrm{mP})$ are compared between the $\mathrm{F}$ and revF stimulation (FmP and $\mathrm{RmP}$ ) and between the naïve and trained ( $\mathrm{N}$ and $\mathrm{Tr}$ ) animal groups. The channel-unique tempMs (tM) of activation evoked by the revF at the location where the F-evoked $\max P$ within the $\mathrm{Al}$ is recorded (RtM at FmP) are compared between the animal groups. B, Coordinate-based Euclidian distances between the F- and the revF-evoked maxP within the Al (FmP-RmP) are compared between the trained $(\mathrm{Tr})$ and naïve $(\mathrm{N})$ animal groups. These distances for the different animal groups are also separately compared with the spontaneous separation distance between the $2 \mathrm{mPs}$ obtained by repeating the $\mathrm{F}$ stimulation twice (1stFmP-2ndFmP; i.e., the internal fluctuation). C, Ratios (or Contrasts) of the revF-evoked maxP relative to the F-evoked $\max P$ within the $\mathrm{Al}(\mathrm{RmP} / \mathrm{FmP})$ are compared between the different animal groups (left). Similarly, the ratios of the revF-evoked tempM at the location of the F-evoked maxP relative to the F-evoked maxP (RtM at FmP/FmP) are compared between the animal groups (right). $\boldsymbol{D}$, Ratios of the F-evoked $\max P$ within the VA relative to the F-evoked $\max P$ within the Al (FmP.VA/FmP) are compared between the naïve $(\mathrm{N})$ and trained $(\mathrm{Tr})$ animal groups. Error bars indicate the standard deviation. ns, not significant. 
Table 2. Comparison between sound onset-to-maximum peak latencies (in $\mathrm{ms}$ ) for different animal groups

\begin{tabular}{|c|c|c|c|c|c|c|}
\hline \multirow{4}{*}{$\begin{array}{l}\text { Sound type } \\
\text { F } \\
\text { revF }\end{array}$} & \multicolumn{4}{|l|}{ Animal group } & \multicolumn{2}{|c|}{$\begin{array}{l}\text { Comparison between } \\
\text { animal groups }\end{array}$} \\
\hline & Trained & Number of hemispheres used & Naïve & Number of hemispheres used & Type of test & $p$ values \\
\hline & $28.3 \pm 5.2$ & 12 & $26.3 \pm 4.5$ & 12 & Welch test & 0.323 \\
\hline & $86.2 \pm 50.2$ & 12 & $111.5 \pm 36.2$ & 12 & Welch test & 0.171 \\
\hline
\end{tabular}

trained animal group, the peaked activation (red/pink domains) evoked by the first $F$ segment was initiated in the dorsal region of the $\mathrm{Al}$ (Fig. $3 \mathrm{C}$, upper panel, small black dot in left frame), shifted ventrally/anteroventrally to the central region of the $\mathrm{Al}$ where it reached the $\max P$ (large black dot), and propagated further ventrally to the VA. However, compared with the trained group, the peaked activation within the VA appeared to be less frequently generated and have smaller peak amplitudes (Fig. 3C, upper panel, small white dot in the left frame). A complete sequence of the activation was shown for another hemisphere in Figure $5 A$. Similar patterns of the Al activation were observed for most of the naïve hemispheres (Fig. 8, right column).

\section{Optical response signals evoked by revF}

Activation patterns evoked by the revF were markedly different from those evoked by the $\mathrm{F}$ in the temporal and spatial dimensions. The revF-evoked activation was generally weak ( $n=15)$ or not evident $(n=3$, namely 2 trained and 1 naïve hemispheres) at the threshold used for the F-evoked activation maps (i.e., 6 SD of the mean of spontaneous activity; Fig. 9). Patterns of the weak activation varied from hemisphere to hemisphere, with some showing dispersed small domains of weak activation (Fig. $3 B$, lower panel) and other showing one major and several minor domains of weak activation (Fig. 3C, lower panel). For the hemispheres in which activation was not evident at the 6 SD threshold (see above), lowering the threshold to the 3 or 4 SD exhibited the pattern of dispersed small activation.

The time courses of activation were also markedly different from those of F-evoked activation. In general, the revF-evoked activation domains did not move systematically from the dorsal to the central region of $\mathrm{Al}$ (Fig. 3B, lower panel) or from the Al to the VA field (Fig. $3 C$, lower panel) during the activation period (Fig. 9). The mean latency from the sound onset to the maxP evoked by the first revF segment within the Al (Fig. 6) was significantly longer than that evoked by the $F$ segment both in the trained (38-186 ms, $86.2 \pm 50.2 \mathrm{~ms}$, SD; $p=6.46 \mathrm{E}-04$; Table 3) and naïve (54-170 ms, $111.5 \pm 36.2 \mathrm{~ms}, \mathrm{SD} ; p=$ 4.84E-08; Table 3) group, with no significant difference between the groups $(p=0.171$; Table 2$)$. Variation in the latency was also significantly greater for the revF stimulation than for the $\mathrm{F}$ stimulation both in the trained $(p=$ 2.31E-0.8; Table 3) and in the naïve groups ( $p=3.07 \mathrm{E}-09$; Table 3).

\section{Spatial segregation of peaked activation and comparisons between animal groups}

We examined the separation distances between the cortical locations of $\mathrm{F}$ - and revF-evoked $\max P$ within the
Al. The coordinate-based mean Euclidian distance was $26.8 \pm 21.3$, SD for the naïve and $33.6 \pm 15.8$, SD for the trained groups (Fig. 7B). Each of these group-unique means of the F-revF peak separation was significantly greater $(p=0.0283$ for the naïve group and $p=0.00071$ for the trained group; Table 4) than the mean spontaneous separation of the two maxPs obtained by repeating the $F$ twice $(9.66 \pm 9.42, \mathrm{SD}$, over seven hemispheres of three trained and four naïve animals). However, these peak-topeak distances were not significantly different between the animal groups (Fig. $7 B, p=0.386$; Table 4).

\section{Comparison of activation between animal groups}

Stimulation with the F segments consistently activated the auditory cortex more strongly than stimulation with the revF segments, with the evoked maxPs (Fig. 7A) being significantly different for both the trained $(0.212 \pm 0.063$, SD vs $0.116 \pm 0.029, \mathrm{SD} ; p=8.74 \mathrm{E}-05$; Table 5$)$ and the naïve groups $(0.199 \pm 0.0600, S D$ vs $114 \pm 0.026, S D ; p$ $=1.97 \mathrm{E}-04$; Table 5). The comparison of these maxPs between the animal groups showed that the F-evoked $\mathrm{Al}$ $\max P$ was slightly larger for the trained group than for the naïve group, but this difference did not reach a significance level (Fig. 7A, $p=0.621$; Table 6). Similarly, the revF-evoked Al maxPs were not statistically different between the animal groups (Fig. 7A, $p=0.872$; Table 6).

\section{Comparison of the activation contrasts between animal groups}

Magnitude of response signals varied substantially among hemispheres; therefore, we normalized the response signals evoked by the revF to those evoked by the $\mathrm{F}$ for each hemisphere. Means of these hemisphereunique contrasts were compared between animal groups. The ratios of the revF-evoked $\max P$ relative to $\mathrm{F}$-evoked $\max P$ within the $\mathrm{Al}(\mathrm{RmP} / \mathrm{FmP})$ were not significantly different between the naïve and trained animal groups $(0.600$ \pm 0.140 , SD vs $0.574 \pm 0.159$, SD, respectively; $p=$ 0.675; Fig. 7C, left; Table 7). However, if the cortical location where stimulus sounds activated the Al maximally (corresponding to the F-evoked maxP) was specifically concerned, the ratio of the revF-evoked tempM at this location relative to the F-evoked $\operatorname{maxP}(\mathrm{RtM}$ at $\mathrm{FmP}$ / $\mathrm{FmP}$ ) was significantly smaller in the trained than in the naïve groups $(0.348 \pm 0.116, S D$ vs $0.449 \pm 0.088, S D$, respectively; $p=0.0264$; Fig. $7 C$, right; Table 7 ), although these revF-evoked tempMs at the F-evoked maxP location themselves were not significantly different between the animal groups $(0.0711 \pm 0.023, S D$ vs $0.0873 \pm$ $0.027, \mathrm{SD}, p=0.129$; Fig. 7A; Table 8). Thus, the reduced contrast for the trained animals indicates that the neuronal population most sensitive to a given natural sound was 
Trained left
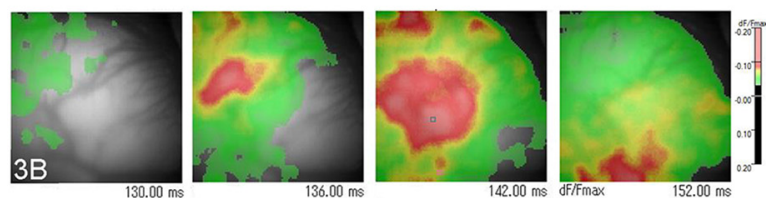

F stimulation
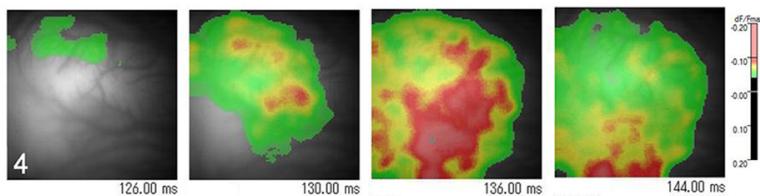

Naïve left
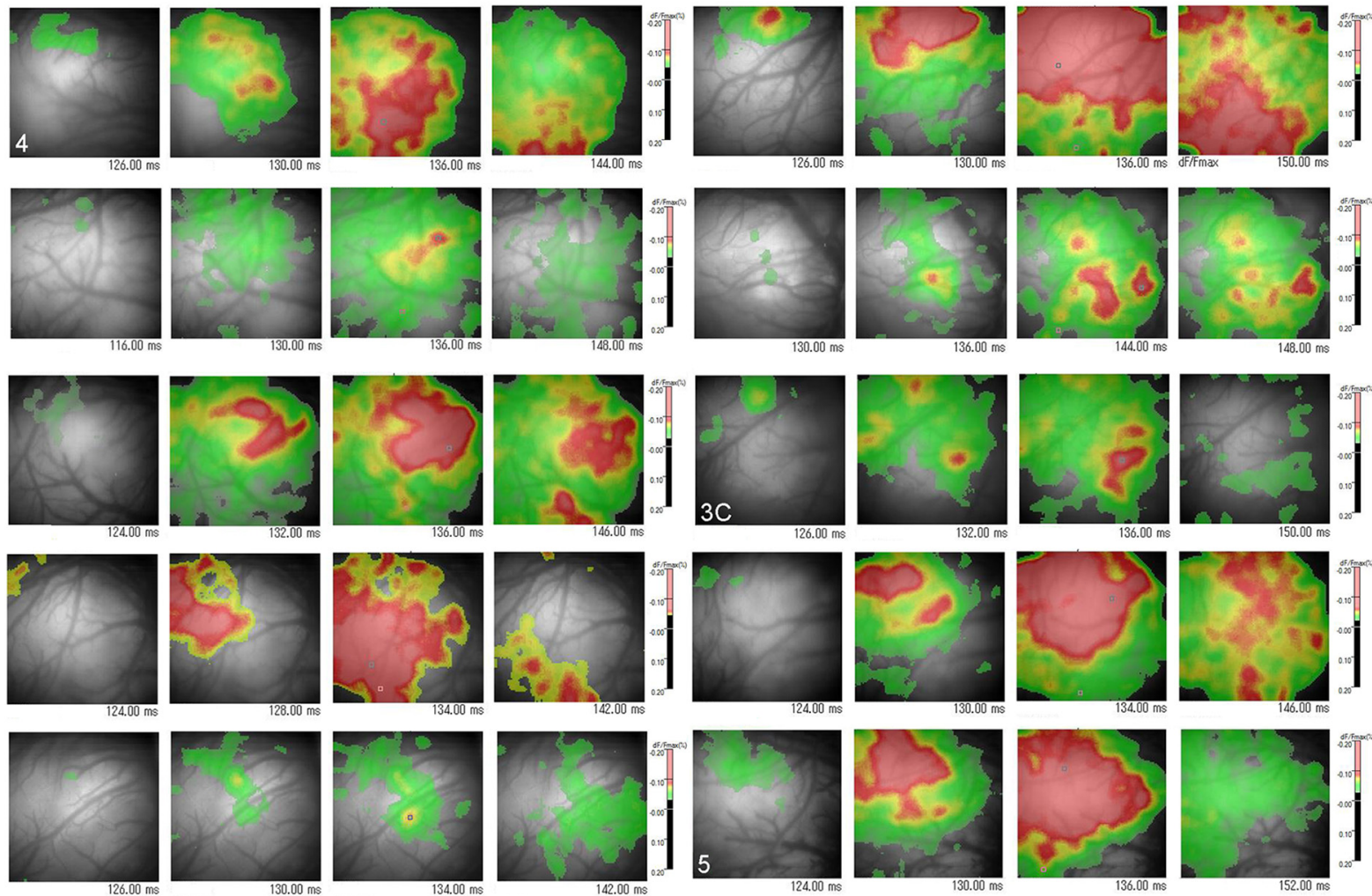

Trained right

Naïve right
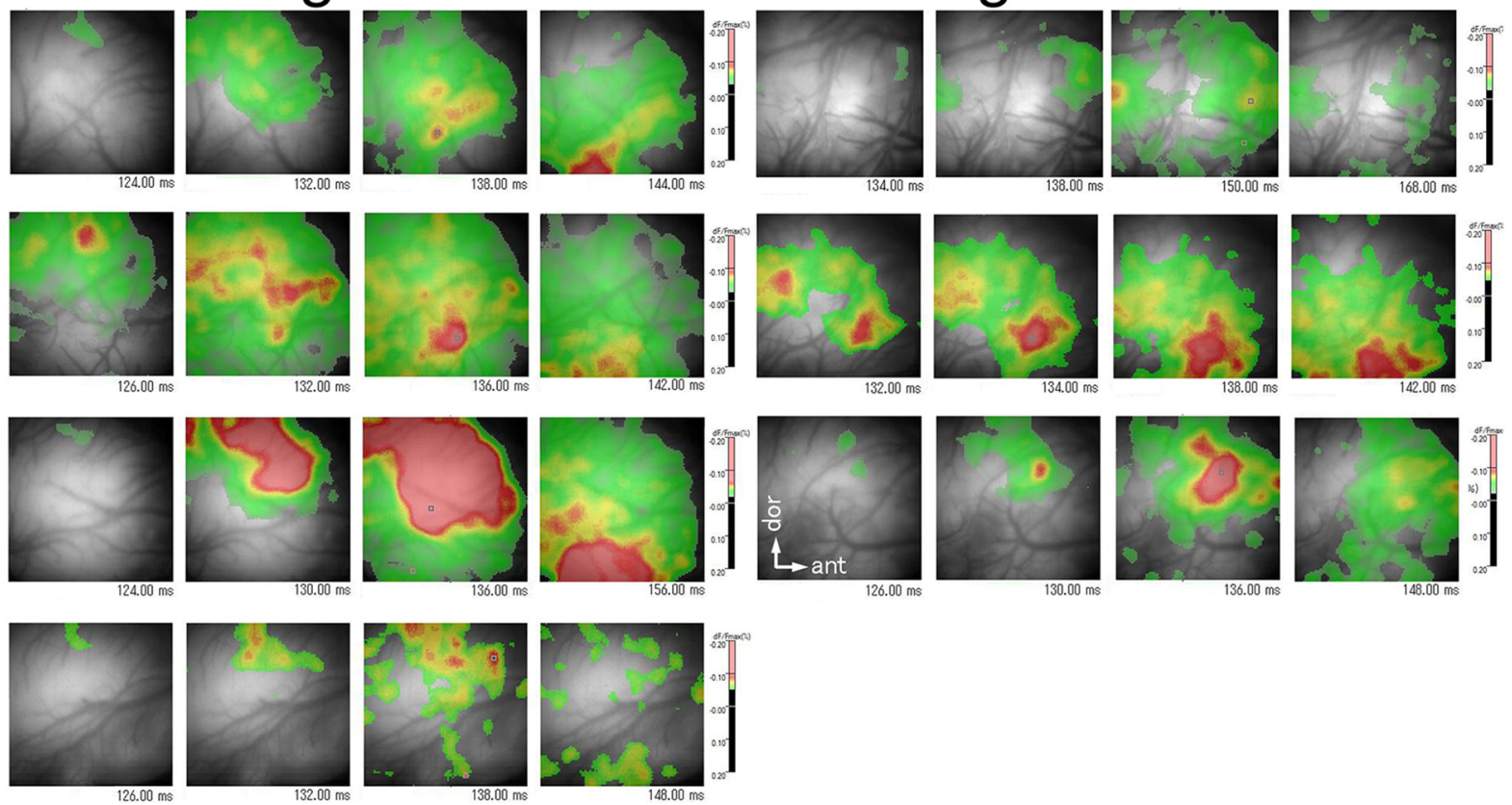

Figure 8. Temporal sequence of the F-evoked activation maps. The activation period during which the F-evoked response signals (dF/Fmax, \%) are above the threshold (6 SD of the mean of spontaneous activity values) is divided into four consecutive phases. The 
continued

activation maps representing each of these phases are chronologically shown (from left to right) in a four-frame panel for a given hemisphere. All hemispheres used for the VA activation analysis are shown. The response signals are color-coded (scale bar, \%) according to their magnitude. All scale bars have a magnitude range of $-0.20-0.20 \%$, and signal values beyond this range are converted to the range maximum, $-0.20 \%$. Domains of the peaked activation (red region) evoked by $\mathrm{F}$ stimulation tend to spread into the ventral one-fifth zone of the image frame (corresponding to the VA) and cross its ventral side more frequently in the trained than in the naïve group (Figure 9). Quantitatively, the maxPs of VA activation normalized to the maxPs of Al activation are significantly larger for the trained than for the naïve group (Fig. 7D). Hemispheres used in other figures are labeled with the white numbers in the left frame of panels. All maps are oriented in the same way (white arrow).

more strongly suppressed by its time-reversed unnatural counterpart after conditioning to the natural version.

\section{Activation of the VA in the trained and naïve groups}

The F-evoked activation peaks first emerged in the dorsal region soon after the sound onset and shifted ventrally to the central Al. The peaked activation then propagated further ventrally/anteroventrally to the VA with minimum attenuation in amplitude for the trained group. Occasionally, the activation propagated further ventrally beyond the ventral edge of the image frame. When the activation was mapped at the threshold larger than the 6 SD of the means of spontaneous activities, the peaked activation was tended to spread to the VA more frequently for the trained (8 out of 10 hemispheres; Fig. 8, left) than for the naïve group (4 out of 8 hemispheres; Fig. 8 , right), though they were not statistically significant (two-tailed, $p$ $=0.321$; Table 9). The F-evoked VA maxPs themselves were not statistically different between the naïve and trained groups $(0.169 \pm 0.074, S D$ vs $0.208 \pm 0.088$, SD; $p=0.1429$; Table 10). However, the normalized maxPs relative to those in Al were significantly different between animal groups; the ratios of the F-evoked VA maxP relative to the F-evoked Al maxP were $0.665 \pm 0.174$ (mean \pm $\mathrm{SD}$ ) for the naïve group versus $0.912 \pm 0.177$ (mean \pm SD) for the trained group ( $p=0.0097$; Fig. $7 D$; Table 10).

In contrast, at the threshold used for the $\mathrm{F}$ activation maps, the revF stimulation did not evoke the peaked activation within the VA $(n=18)$ for either animal group (Fig. 9, data not statistically analyzed).

\section{Discussion}

\section{Cortical activation evoked by temporally asymmetric sound pairs}

The trial-unique $\operatorname{maxPs}$ of $\mathrm{Al}$ activation were significantly smaller for revF than for $F$ stimulation (Fig. 7), indicating that the temporal asymmetry of envelopes affects the synaptic excitation. In addition to the magnitude difference, the latency to the $\max P$ was prolonged with a greater intertrial fluctuation for revF than for $F$ stimulation (Fig. 6). This response asymmetry may be due to the envelope transition effects on onset responses (Heil, 1997a,b). The response magnitude and latency are functions of the first derivative of the rise-time transition to peak pressure. Thus, the discharge rate is higher when the instantaneous onset slope of envelope is steeper and the first-spike latency is shortened when the acceleration of peak pressure is increased. Ramped-down sounds generated by applying convulsive force against hard materials, like the $\mathrm{F}$ of the present study, are characterized by a quick rise and slow decay of the envelope, while ramped-up sounds, such as the revF, are characterized by the opposite envelope configuration. Therefore, it is likely that the revF evokes lower spike discharge rates and longer peak latencies than the F.

The stimulus asymmetry was also reflected by the location of the maxPs in the Al. The discrete maxPs of activation evoked by $\mathrm{F}$ and revF stimulation were separated more widely than the two maxPs evoked by repeating the $\mathrm{F}$ twice were segregated (Fig. 7B). This significant separation of the two maxPs indicates that a neuronal population activated maximally by the normal forward sound is not the population that is activated maximally by its reversed counterpart despite their identical spectrum content.

The propagation of activation from the dorsal to ventral regions of the $\mathrm{Al}$, as revealed by $\mathrm{F}$ stimulation, was not fully reproduced by revF stimulation. The so-called isofrequency axis is oriented dorsoventrally, roughly perpendicular to the anterocaudal frequency gradient in the Al of the guinea pig (Hellweg et al., 1977; Redies et al., 1989). It is known that Al pyramidal neurons located along the isofrequency axis are interconnected through their horizontal collaterals (Ojima et al., 1991). Thus, the absence of the dorsoventral propagation of activation by the revF stimulation may be a reflection of weak or no interactions between Al cortical neurons through their intrinsic horizontal connections. An alternative interpretation is also possible. Our results may reflect disturbance of gradually delaying thalamocortical transmission along the Al isofrequency axis (Taniguchi et al., 1992; Wallace et al., 2000; Nishimura et al., 2007). Regardless, either hypothesis would lead to a deteriorated temporal sequence of activation peaks across the Al.

\section{Modification after conditioning to wideband natural sounds}

Learning did not significantly affect the magnitude of activation peaks within the Al (Fig. 7A). Naïve animals had never been exposed to either the $\mathrm{F}$ or revF before testing, whereas trained animals had been intensively exposed to the $\mathrm{F}$. Despite this difference in experience, the $\operatorname{maxPs}$ of F- and revF-evoked activation were almost the same whether animals were trained or not. This finding appears to contradict the well-known frequency-specific plasticity, but suggests that the learning of wideband sounds, such as the present $F$, may not augment the responsiveness to particular frequencies, probably because multiple frequency components would be used as spectral cues during conditioning. Instead, learning increased the con- 
Trained left
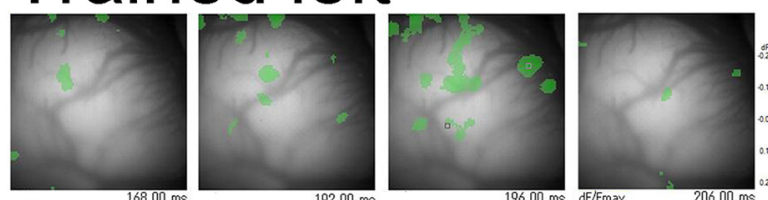

\section{revF stimulation}
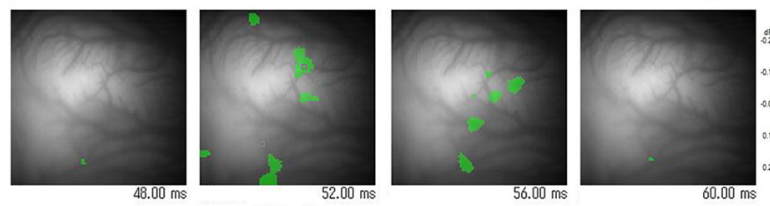

Naïve left
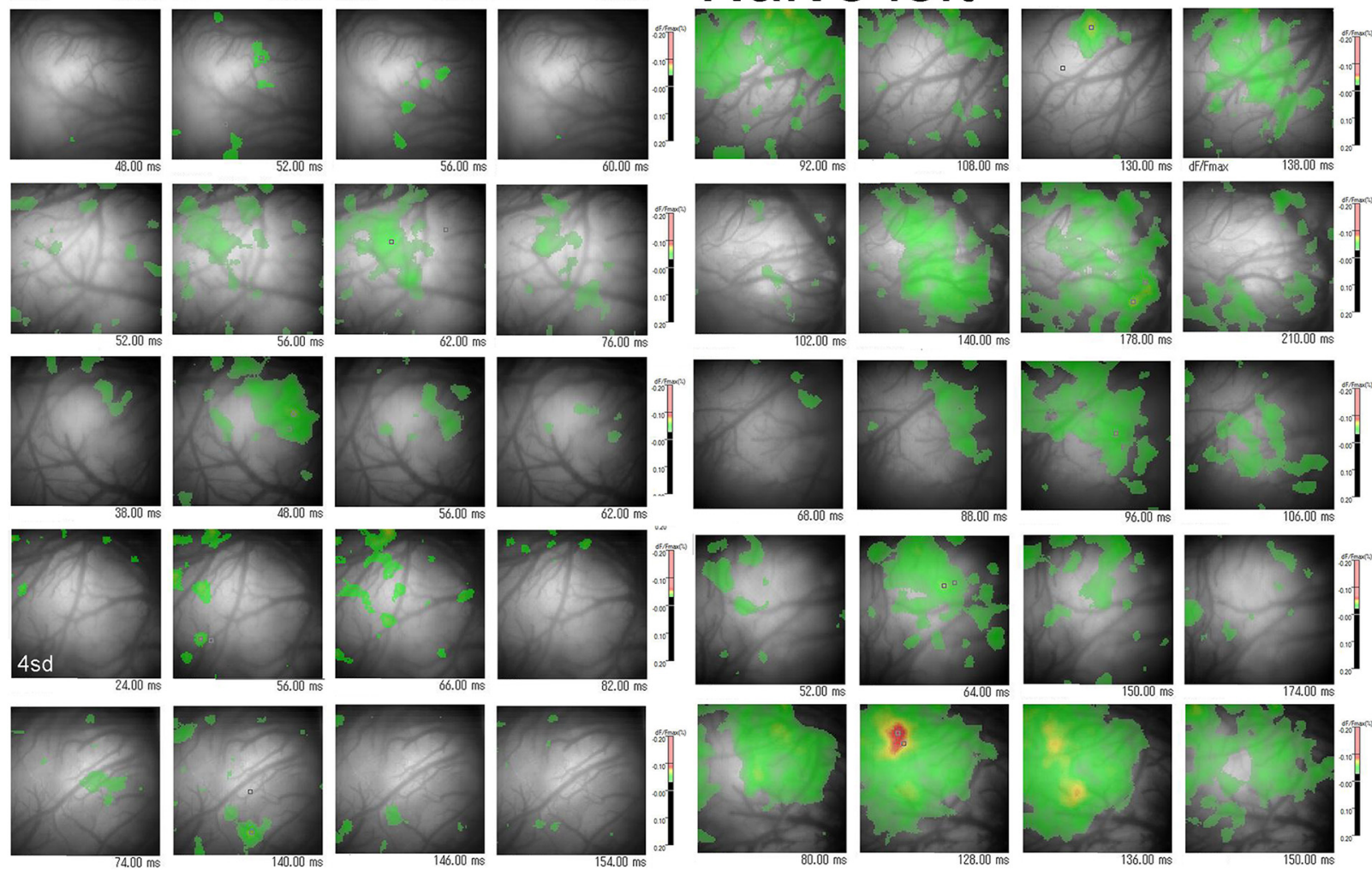

Trained right
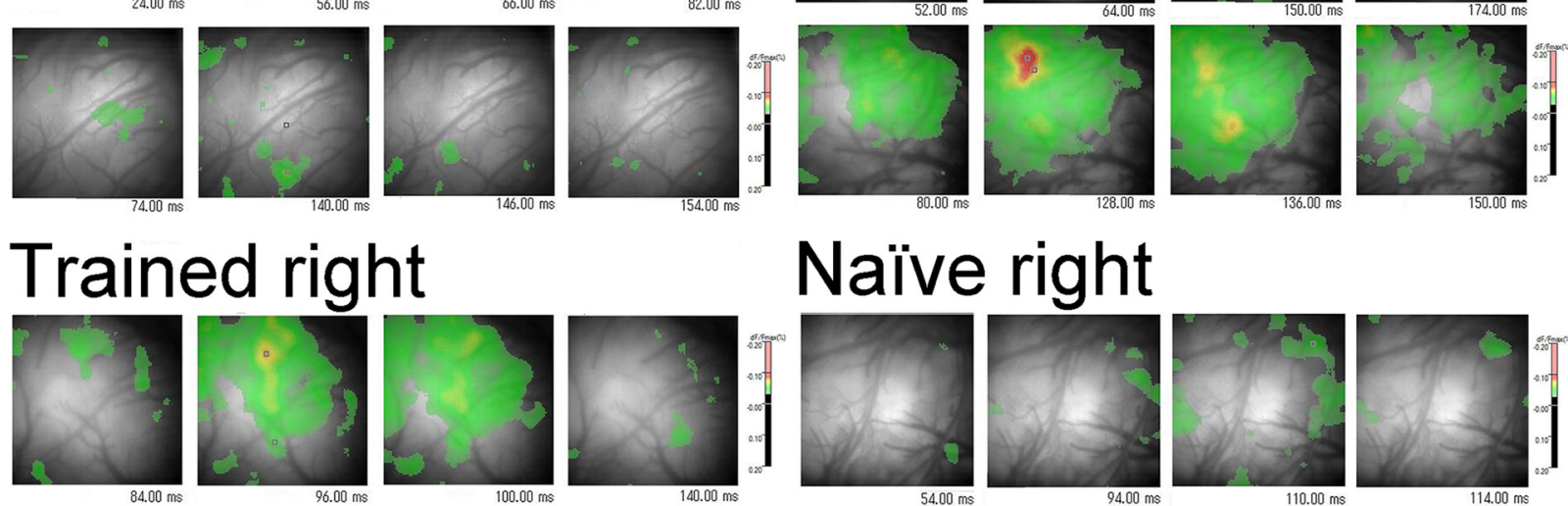

Naïve right
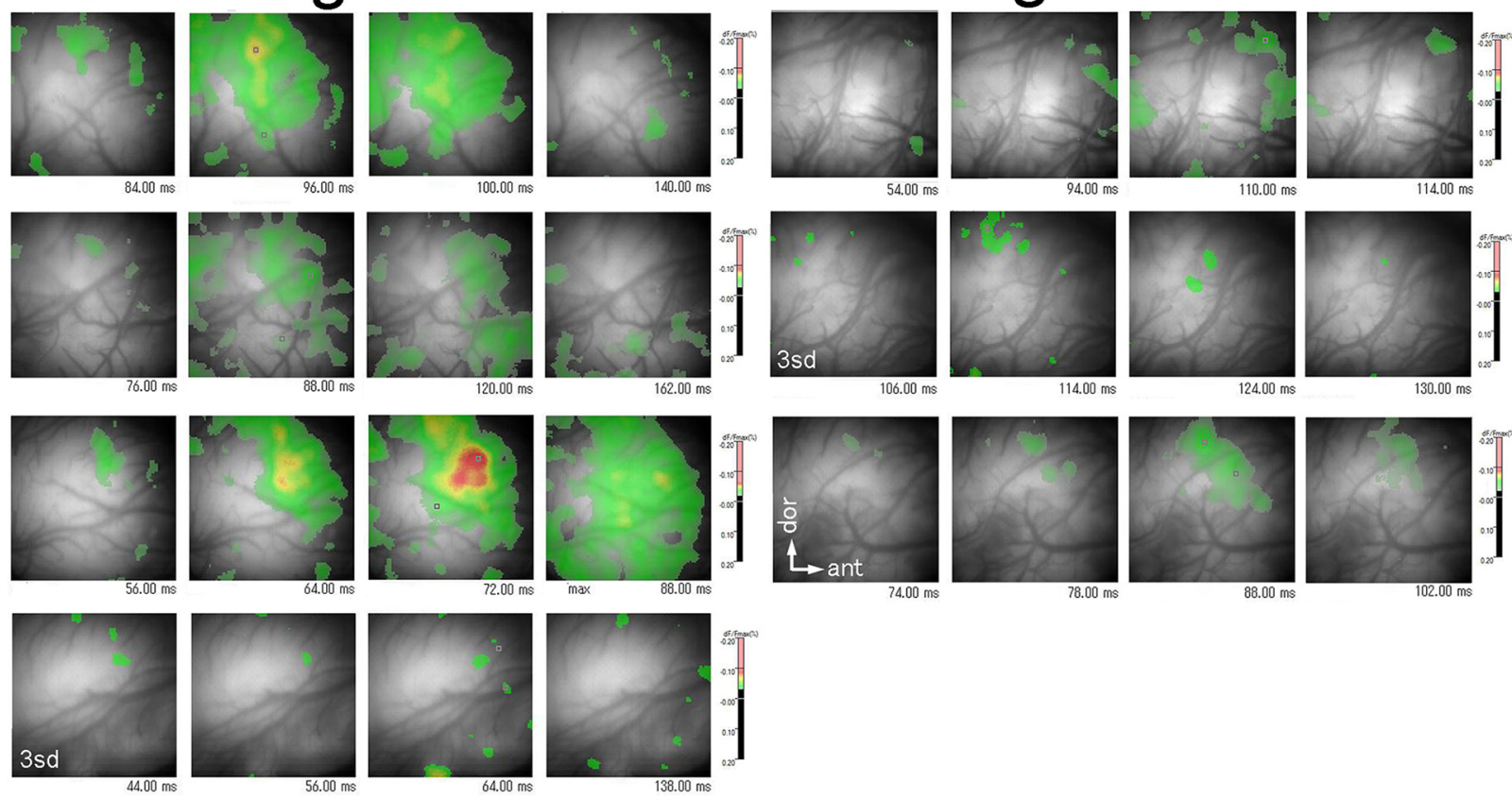

Figure 9. Temporal sequence of the revF-evoked activation maps. The activation period during which the revF-evoked response signals (dF/Fmax, \%) are above the threshold (6 SD of the mean of the spontaneous activity values, but the 4 or 3 SD for some 


\section{continued}

hemispheres as labeled directly) is divided into four consecutive phases. The hemisphere-unique activation maps representing each of these phases are chronologically arranged (from left to right) in a four-frame panel for a given hemisphere. All hemispheres used for the VA activation analysis are shown. The response signals are color-coded (scale bar, \%) according to their magnitude. All scale bars have a magnitude range of $-0.20-0.20 \%$. Activation evoked by the revF stimulation is generally weak except for three hemispheres that have the peaked activation (small red domains) within the Al. However, none of the revF-stimulation evokes the peaked activation within the VA. The panels at the corresponding location of Figures 8 and 9 are of the same hemisphere. All maps are oriented in the same way (see white arrows).

trast of synaptic responses evoked at the Al location that was activated maximally by the temporally asymmetric sound pair. It implies that when the neuronal population that is maximally activated by a forward sound is more vigorously suppressed by its asymmetric counterpart after learning (Fig. 7C). Conditioning to a particular temporal structure modifies network to be tuned preferentially to that envelope, and this modified network is now more resistant to be activated by the asymmetrically degraded temporal structure.

\section{Activation spread into the VA}

Time course of the activation maps showed that the F-evoked activation extended from the dorsal to central region of the $\mathrm{Al}$ and then propagated to the VA (Figs. 3B, $4 A)$. This propagation of F-evoked activation to the VA occurred more robustly after training, resulting in the greater VA-to-Al peak activation ratios for the trained animal group (Fig. $7 D$ ). Note that since the naïve animals had never heard the stimulus sound before testing, this VA activation might possibly be facilitated simply by the repeated exposure during training but not by learning. We cannot explicitly differentiate these possibilities. However, the finding that VA activation could be sporadically observed even in the naïve animals (Fig. 8) and also by pure tones stimulation in our routine mapping experiments (Horikawa et al., 2001) suggests that neither of the above possibilities may be a critical requisite for induction of the

Table 3. Comparison of sound onset-to-maximum peak latencies (in $\mathrm{ms}$ ) and their variations between different sound types

\begin{tabular}{|c|c|c|c|c|c|c|c|}
\hline \multirow{3}{*}{$\begin{array}{l}\text { Animal } \\
\text { group } \\
\text { Trained }\end{array}$} & \multirow{3}{*}{$\begin{array}{l}\text { Number of } \\
\text { hemispheres used } \\
12\end{array}$} & \multicolumn{2}{|l|}{ Sound type } & \multicolumn{2}{|c|}{$\begin{array}{l}\text { Comparison of } \\
\text { means between } \\
\text { sound types }\end{array}$} & \multicolumn{2}{|c|}{$\begin{array}{l}\text { Comparison of } \\
\text { variations between } \\
\text { sound types }\end{array}$} \\
\hline & & $\mathrm{F}($ mean $\pm \mathrm{SD})$ & $\operatorname{revF}($ mean $\pm S D)$ & Type of test & $p$ values & Type of test & $p$ values \\
\hline & & $28.3 \pm 5.2$ & $86.2 \pm 50.2$ & $t$ test & $6.46 \mathrm{E}-04$ & F-test & $2.31 \mathrm{E}-08$ \\
\hline Naïve & 12 & $26.3 \pm 4.5$ & $111.5 \pm 36.2$ & $t$ test & $4.84 \mathrm{E}-08$ & F-test & 3.07E-09 \\
\hline
\end{tabular}

Table 4. Comparison between separation distance of maxPs evoked by sounds and internal fluctuation distance

\begin{tabular}{|c|c|c|c|c|c|c|c|c|}
\hline \multirow{2}{*}{$\begin{array}{l}\text { Animal } \\
\text { group } \\
\text { Trained }\end{array}$} & \multicolumn{2}{|c|}{$\begin{array}{l}\text { Euclidian distance between } \\
\text { F- and revF-evoked maxPs } \\
\text { of activation }\end{array}$} & \multicolumn{2}{|c|}{$\begin{array}{l}\text { Internal fluctuation, } \\
\text { distance of maxPs } \\
\text { evoked by repeating F }\end{array}$} & \multicolumn{2}{|c|}{$\begin{array}{l}\text { Comparison of } \\
\text { separation distance } \\
\text { of stimulus-evoked } \\
\text { maxPs to internal } \\
\text { fluctuation distance }\end{array}$} & \multicolumn{2}{|c|}{$\begin{array}{l}\text { Comparison of separation } \\
\text { distance of F-evoked and } \\
\text { revF-evoked maxPs } \\
\text { between animal groups }\end{array}$} \\
\hline & $\begin{array}{l}\text { Coordinate-based } \\
\text { distance }\end{array}$ & $\begin{array}{l}\text { Number of } \\
\text { hemisphere } \\
\text { s used }\end{array}$ & $\begin{array}{l}\text { Coordinate-based } \\
\text { distance }\end{array}$ & $\begin{array}{l}\text { Number of } \\
\text { hemispheres } \\
\text { used }\end{array}$ & Type of test & $p$ values & Type of test & $p$ value \\
\hline
\end{tabular}

Table 5. Comparison between magnitudes of maxPs evoked by $\mathrm{F}$ stimulation and revF stimulation, dF/Fmax \%

\begin{tabular}{|c|c|c|c|c|c|}
\hline Animal group & $\begin{array}{l}\text { Number of } \\
\text { hemispheres used }\end{array}$ & \multicolumn{2}{|c|}{$\begin{array}{l}\text { MaxPs of activation evoked by } \\
\mathrm{F} \text { and revF stimulation }\end{array}$} & \multicolumn{2}{|c|}{$\begin{array}{l}\text { Comparison between } \\
\text { sound types }\end{array}$} \\
\hline Trained & 12 & $0.212 \pm 0.063$ & $0.116 \pm 0.029$ & $t$ test & $8.74 \mathrm{E}-05$ \\
\hline
\end{tabular}

Table 6. Comparison between maxPs of activation for different animal groups, dF/Fmax \%

\begin{tabular}{|c|c|c|c|c|c|c|}
\hline \multirow{4}{*}{$\begin{array}{l}\text { Sound type } \\
\text { F } \\
\text { revF }\end{array}$} & \multirow{2}{*}{ Animal group } & \multirow[b]{2}{*}{$\begin{array}{l}\text { Number of } \\
\text { hemispheres used }\end{array}$} & \multirow[b]{2}{*}{ Naïve } & \multirow[b]{2}{*}{$\begin{array}{l}\text { Number of } \\
\text { hemispheres used }\end{array}$} & \multicolumn{2}{|c|}{$\begin{array}{l}\text { Comparison between } \\
\text { animal groups }\end{array}$} \\
\hline & & & & & Type of test & $p$ values \\
\hline & $0.212 \pm 0.063$ & 12 & $0.199 \pm 0.060$ & 12 & Welch test & 0.621 \\
\hline & $0.116 \pm 0.029$ & 12 & $0.114 \pm 0.026$ & 12 & Welch test & 0.872 \\
\hline
\end{tabular}


Table 7. Comparison between contrasts of revF-activation relative to F-evoked activation for different animal groups

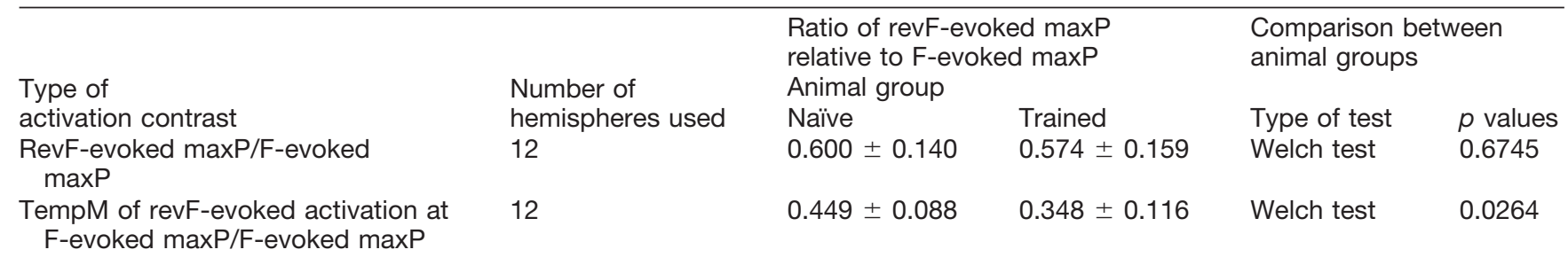

robust VA activation. We rather claim involvement of the emotive valence of training itself. During the competitive training, animals competed each other for food and frequently blocked the competitor to access food (Ojima and Horikawa, 2016), thus being in a strongly aggressive state. Aggression is known to be a highly emotional state (Albert et al., 1989; Nelson and Trainor, 2007). Therefore, it is expected that the neural network of emotion together with the reward system would be activated during the conditioning. Emotional learning is mediated through the corticoamygdala pathway (LeDoux, 1992; Ono et al., 1995; Phelps and LeDoux, 2005). Anatomically, the primary sensory cortex generally does not project to the amygdala, while the higher association cortices, via primary-to-association corticocortical connections, project to the amygdala (Grosso et al., 2015). For rats, networks for this processing include the following auditory connections (Romanski and LeDoux, 1993); the TE1 (corresponding to the $\mathrm{Al}$ ) projects to the area just ventral to it (TE1v) and to other fields further surrounding the TE1v (i.e., TE2c and TE3v), and these nonprimary fields give rise to the corticoamygdala projection. Although no comparative data have been available, the VA of the guinea pig is possibly homologous to the TE1v on the basis of its topographical and connectional relationships with the $\mathrm{Al}$ (Wallace et al., 2002). The comparisons of activation strength between the trained and naïve animals revealed the stronger F-evoked VA activation after the competitive training (Fig. 7D). Altogether, the enhanced emotional state, the possible homology of the VA to rat not-Als and their connections to the amygdala suggest intriguing implications for the role of emotional state and amygdala in perceptional leaning.

\section{Comparison to the previous studies}

On the basis of spike counts, comparisons of neuronal activation evoked by natural calls and their time-reversed versions have been made for bird songs (McCasland and Konishi, 1981; Margoliash, 1983), marmoset twitter calls (Wang et al., 1995; Wang and Kadia, 2001) and cat meow calls (Gehr et al., 2000). Comparisons were also made between the artificial sinusoids of temporally asymmetric envelopes (Lu et al., 2001a,b; Wang et al., 2014). Interestingly, the conclusions from the studies using the natural and artificial sound types were not consistent. For animal songs/calls, neurons were generally more respon-

Table 8. Comparison of tempMs of revF-evoked activation recorded at the location of F-evoked maxP between different animal groups, dF/Fmax \%

\begin{tabular}{llll}
\hline & Number of & Comparison between \\
Animal group & hemispheres used & RevF-evoked tempM & $\begin{array}{l}\text { animal groups } \\
\text { at F-evoked maxP }\end{array}$ \\
Trained & 12 & $0.0711 \pm 0.023$ & Type of test \\
Naïve & 12 & $0.0873 \pm 0.027$ & Welch test \\
\hline
\end{tabular}

Table 9. Comparison of occurrence of VA activation peaks between animal groups

\begin{tabular}{|c|c|c|c|c|c|}
\hline Animal group & $\begin{array}{l}\text { Number of } \\
\text { hemispheres } \\
\text { used }\end{array}$ & $\begin{array}{l}\text { Number of hemispheres } \\
\text { with VA peaked } \\
\text { activation }\end{array}$ & $\begin{array}{l}\text { Number of hemispheres } \\
\text { without VA peaked } \\
\text { activation }\end{array}$ & $\begin{array}{l}\text { Comparison between } \\
\text { animal groups } \\
\text { Type of test }\end{array}$ & $p$ value \\
\hline Naïve & 8 & 4 & 4 & Fisher's exact probability & 0.3213 \\
\hline
\end{tabular}

Table 10. Comparisons of F-evoked maxPs in the VA and Al between trained and naïve animal groups

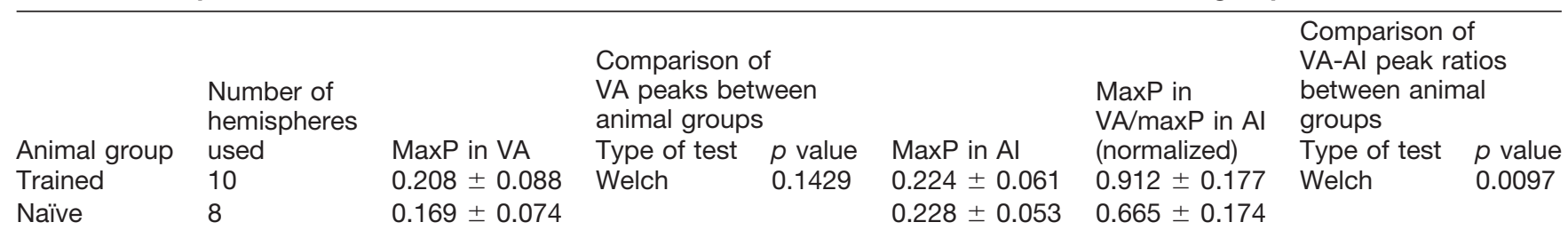


sive to the natural versions than their time-reversed counterparts whether animals were anaesthetized (Wang et al., 1995; Gehr et al., 2000) or awake (McCasland and Konishi, 1981; Margoliash, 1983). Thus, the differences in the response magnitude may reflect differences in innate network or past experience. For the artificial sounds, a larger proportion of neurons preferred ramped-up sinusoids to ramped-down sinusoids (Lu et al., 2001b; Wang et al., 2014). This response preference was apparently opposite to our present result, which demonstrated a stronger activation with the ramped-down $\mathrm{F}$ than with its timereversed revF. However, when we closely inspected the relevant responses evoked by the stimuli that were comparable in duration and envelope shape to ours and presented in the similar manner (see Figure 4 in Wang et al., 2014), the ramped-down sinusoids appear to generate larger responses than the counterparts if the discharge rates are measured only during the sound-on period comparable to ours (i.e., $80 \mathrm{~ms}$ ).

Voltage-sensitive dye signals more likely reflect local field potentials (LFPs) than spiking of single neurons. Fractional changes in light intensity (dF/Fmax) are in proportional to changes in the membrane potential averaged across all neural elements under individual sensors (Chemla and Chavane, 2010). Their signal-to-noise ratio, however, is generally worse than that of the LFPs (London et al., 1989; Prechtl et al., 1997), since optical signals depend on many biological and technical factors. The $\gamma$-band oscillations in LFPs are stimulus dependent and engaged in coding sensory objects (Herrmann et al., 2010). In the auditory domain, presentations of single clicks, pure tones, and amplitude-modulated sounds generally evoke $\gamma$-band oscillations in a phase-locked manner (Galambos et al., 1981; Pantev et al., 1991; Franowicz and Barth, 1995). In addition, natural sounds including human voices (Crone et al., 2006) and animal calls (Medvedev and Kanwal, 2004) have been reported to evoke $\gamma$-band oscillations depending on the meaningfulness of those sounds. Thus, when communication calls were temporally reversed, the evoked $\gamma$-band oscillations were generally attenuated, as shown for the bat communication calls (Medvedev and Kanwal, 2008). Like LFPs, the optical signals of voltage-sensitive dyes report the population activity of neural elements, but only low frequency oscillations of optical signals (around $10 \mathrm{~Hz}$ ) have so far been observed in a limited number of studies in vivo, for example, from the turtle olfactory bulb (Lam et al., 2000) and also from the rat visual and turtle somatosensory cortices (London et al., 1989; Prechtl et al., 1997). In contrast to these recordings, our sound-evoked optical signals, band-passed at a frequency range of $4.0-40 \mathrm{~Hz}$ (overlapped partially with the typical $30-$ to $80-\mathrm{Hz} \gamma$-band range) did not exhibit prominent oscillating components that had been previously found within the LFPs of the Al. Preliminary measurements confirmed this by filtering the unfiltered original signal at the above-mentioned typical $\gamma$-band range and revealed a few cycles of fluctuation that were very small in amplitude and strictly coincident with the slow-wave large response. For now, it is unclear why $\gamma$-band components could not be observed in our optical signals. Further examination will be needed to interpret this apparent absence of auditory oscillatory components in the optical signals.

We assume that the activation patterns of voltagesensitive dye signals reflect the network's processing of instantaneous spectral content within sounds, probably because of the relatively slow response times. For the network to perform such rapid processing, different populations of neurons must be continuously recruited because of their refractory periods. This rapid recruitment of different neuronal populations may be reflected in the propagation of activation along the $\mathrm{Al}$ iso-frequency axis that likely represent temporally varying spectra within complex sounds (Langner et al., 2009; Brewer and Barton, 2016).

\section{References}

Akeroyd MA, Patterson RD (1995) Discrimination of wideband noises modulated by a temporally asymmetric function. J Acoust Soc Am 98:2466-2474. CrossRef

Albert DJ, Petrovic DM, Walsh ML (1989) Competitive experience activates testosterone-dependent social aggression toward unfamiliar males. Physiol Behav 45:723-727. Medline

Berryman JC (1976) Guinea-pig vocalizations: their structure, causation and function. Z Tierpsychol 41:80-106. Medline

Brewer AA, Barton B (2016) Maps of the auditory cortex. Annu Rev Neurosci 39:385-407. CrossRef Medline

Chemla S, Chavane F (2010) Voltage-sensitive dye imaging: technique review and models. J Physiol Paris 104:40-50. CrossRef Medline

Cohen LB, Salzberg BM, Grinvald A (1978) Optical methods for monitoring neuron activity. Annu Rev Neurosci 1:171-182. CrossRef Medline

Crone NE, Sinai A, Korzeniewska A (2006) High-frequency gamma oscillations and human brain mapping with electrocorticography. Prog Brain Res 159:275-295. CrossRef Medline

Doupe AJ, Konishi M (1991) Song-selective auditory circuits in the vocal control system of the zebra finch. Proc Natl Acad Sci USA 88:11339-11343. Medline

Esser KH, Condon CJ, Suga N, Kanwal JS (1997) Syntax processing by auditory cortical neurons in the FM-FM area of the mustached bat Pteronotus parnellii. Proc Natl Acad Sci USA 94:14019-14024. Medline

Fay RR, Chronopoulos M, Patterson RD (1996) The sound of a sinusoid: perception and neural representations in the goldfish (Carassius auratus). Audit Neurosci 2:377-392.

Franowicz MN, Barth DS (1995) Comparison of evoked potentials and high-frequency (gamma-band) oscillating potentials in rat auditory cortex. J Neurophysiol 74:96-112. Medline

Galambos R, Makeig S, Talmachoff PJ (1981) A 40-Hz auditory potential recorded from the human scalp. Proc Natl Acad Sci USA 78:2643-2647. Medline

Gehr DD, Komiya H, Eggermont JJ (2000) Neuronal responses in cat primary auditory cortex to natural and altered species-specific calls. Hear Res 150:27-42. Medline

Ghazanfar AA, Smith-Rohrberg D, Hauser MD (2001) The role of temporal cues in rhesus monkey vocal recognition: orienting asymmetries to reversed calls. Brain Behav Evol 58:163-172. CrossRef Medline

Grosso A, Cambiaghi M, Concina G, Sacco T, Sacchetti B (2015) Auditory cortex involvement in emotional learning and memory. Neuroscience 299:45-55. CrossRef Medline

Heil P (1997a) Auditory cortical onset responses revisited. I. Firstspike timing. J Neurophysiol 77:2616-2641. Medline

Heil P (1997b) Auditory cortical onset responses revisited. II. Response strength. J Neurophysiol 77:2642-2660. Medline 
Hellweg FC, Koch R, Vollrath M (1977) Representation of the cochlea in the neocortex of guinea pigs. Exp Brain Res 29:467-474. Medline

Herrmann CS, Fründ I, Lenz D (2010) Human gamma-band activity: a review on cognitive and behavioral correlates and network models. Neurosci Biobehav Rev 34:981-992. CrossRef Medline

Horikawa J, Hess A, Nasu M, Hosokawa Y, Scheich H, Taniguchi I (2001) Optical imaging of neural activity in multiple auditory cortical fields of guinea pigs. Neuroreport 12:3335-3339. Medline

Horikawa J, Hosokawa Y, Kubota M, Nasu M, Taniguchi I (1996) Optical imaging of spatiotemporal patterns of glutamatergic excitation and GABAergic inhibition in the guinea-pig auditory cortex in vivo. J Physiol 497:629-638. CrossRef

Irino T, Patterson RD (1996) Temporal asymmetry in the auditory system. J Acoust Soc Am 99:2316-2331. CrossRef

Lam YW, Cohen LB, Wachowiak M, Zochowski MR (2000) Odors elicit three different oscillations in the turtle olfactory bulb. J Neurosci 20:749-762. Medline

Langner G, Dinse HR, Godde B (2009) A map of periodicity orthogonal to frequency representation in the cat auditory cortex. Front Integr Neurosci 3:27. CrossRef Medline

LeDoux JE (1992) Brain mechanisms of emotion and emotional learning. Curr Opin Neurobiol 2:191-197. Medline

Le Prell CG, Moody DB (2000) Factors influencing the salience of temporal cues in the discrimination of synthetic Japanese monkey (Macaca fuscata) coo calls. J Exp Psychol Anim Behav Process 26:261-273. CrossRef

Licklider JCR, Miller GA (1951) The perception of speech. In: Handbook of experimental psychology (Stevens SS, ed), pp 1040-1074. New York: Wiley.

London JA, Cohen LB, Wu JY (1989) Optical recordings of the cortical response to whisker stimulation before and after the addition of an epileptogenic agent. J Neurosci 9:2182-2190. Medline

Lu T, Liang L, Wang X (2001a) Temporal and rate representations of time-varying signals in the auditory cortex of awake primates. Nat Neurosci 4:1131-1138. CrossRef Medline

Lu T, Liang L, Wang X (2001b) Neural representations of temporally asymmetric stimuli in the auditory cortex of awake primates. $\mathrm{J}$ Neurophysiol 85:2364-2380. Medline

Margoliash D (1983) Acoustic parameters underlying the responses of song-specific neurons in the white-crowned sparrow. J Neurosci 3:1039-1057. Medline

McCasland JS, Konishi M (1981) Interaction between auditory and motor activities in an avian song control nucleus. Proc Natl Acad Sci USA 78:7815-7819. Medline

Medvedev AV, Kanwal JS (2004) Local field potentials and spiking activity in the primary auditory cortex in response to social calls. $J$ Neurophysiol 92:52-65. CrossRef Medline

Medvedev AV, Kanwal JS (2008) Communication call-evoked gamma-band activity in the auditory cortex of awake bats is modified by complex acoustic features. Brain Res 1188:76-86. CrossRef Medline

Nelson RJ, Trainor BC (2007) Neural mechanisms of aggression. Nat Rev Neurosci 8:536-546. CrossRef Medline

Nishimura M, Shirasawa H, Kaizo H, Song WJ (2007) New field with tonotopic organization in guinea pig auditory cortex. J Neurophysiol 97:927-932. CrossRef Medline

Ojima H, Horikawa J (2016) Recognition of modified conditioning sounds by competitively trained guinea pigs. Front Behav Neurosci 9:373. CrossRef Medline
Ojima H, Honda CN, Jones EG (1991) Patterns of axon collateralization of identified supragranular pyramidal neurons in the cat auditory cortex. Cereb Cortex 1:80-94. CrossRef

Ojima H, Taira M, Kubota M, Horikawa J (2012) Recognition of non-harmonic natural sounds by small mammals using competitive training. PLoS One 7:e51318. CrossRef Medline

Ono T, Nishijo H, Uwano T (1995) Amygdala role in conditioned associative learning. Prog Neurobiol 46:401-422. Medline

Pantev C, Makeig S, Hoke M, Galambos R, Hampson S, Gallen C (1991) Human auditory evoked gamma-band magnetic fields. Proc Natl Acad Sci USA 88:8996-9000. Medline

Patterson RD (1994a) The sound of a sinusoid: spectral models. $J$ Acoust Soc Am 96:1409-1418.

Patterson RD (1994b) The sound of a sinusoid: time-interval models. J Acoust Soc Am 96:1419-1428.

Phelps EA, LeDoux JE (2005) Contributions of the amygdala to emotion processing: from animal models to human behavior. Neuron 48:175-187. CrossRef Medline

Prechtl JC, Cohen LB, Pesaran B, Mitra PP, Kleinfeld D (1997) Visual stimuli induce waves of electrical activity in turtle cortex. Proc Natl Acad Sci USA 94:7621-7626. Medline

Recanzone GH (2008) Representation of con-specific vocalizations in the core and belt areas of the auditory cortex in the alert macaque monkey. J Neurosci 28:13184-13193. CrossRef Medline

Redies H, Sieben U, Creutzfeldt OD (1989) Functional subdivisions in the auditory cortex of the guinea pig. J Comp Neur 282:473-488. CrossRef Medline

Romanski LM, LeDoux JE (1993) Information cascade from primary auditory cortex to the amygdala: corticocortical and corticoamygdaloid projections of temporal cortex in the rat. Cereb Cortex 6:515-532. Medline

Saberi K, Perrott DR (1999) Cognitive restoration of reversed speech. Nature 398:760. CrossRef Medline

Schlauch RS, Ries DT, DiGiovanni JJ (2001) Duration discrimination and subjective duration for ramped and damped sounds. J Acoust Soc Am 109:2880-2887. Medline

Taniguchi I, Horikawa J, Moriyama T, Nasu M (1992) Spatio-temporal pattern of frequency representation in the auditory cortex of guinea pigs. Neurosci Lett 146:37-40. Medline

Wallace MN, Palmer AR (2009) Functional subdivisions in lowfrequency primary auditory cortex (Al). Exp Brain Res 194:395408. CrossRef Medline

Wallace MN, Rutkowski RG, Palmer AR (1999) A ventrorostral belt is adjacent to the guinea pig primary auditory cortex. Neuroreport 10:2095-2099. Medline

Wallace MN, Rutkowski RG, Palmer AR (2000) Identification and localisation of auditory areas in guinea pig cortex. Exp Brain Res 132:445-456. Medline

Wallace MN, Rutkowski RG, Palmer AR (2002) Interconnections of auditory areas in the guinea pig neocortex. Exp Brain Res 143: 106-119. CrossRef Medline

Wang X, Kadia SC (2001) Differential representation of speciesspecific primate vocalizations in the auditory cortices of marmoset and cat. J Neurophysiol 86:2616-2620.

Wang X, Merzenich MM, Beitel R, Schreiner CE (1995) Representation of a species-specific vocalization in the primary auditory cortex of the common marmoset: temporal and spectral characteristics. J Neurophysiol 74:2685-2706. Medline

Wang J, Qin L, Chimoto S, Tazunoki S, Sato Y (2014) Response characteristics of primary auditory cortex neurons underlying perceptual asymmetry of ramped-up and damped sounds. Neuroscience 256:309-321. CrossRef 